\title{
MARTIN ANDLER
}

\section{Ordre de Bruhat et transfert : le cas réel}

Bulletin de la S. M. F., tome 122, no 1 (1994), p. 119-145

<http://www.numdam.org/item?id=BSMF_1994_122_1_119_0>

(C) Bulletin de la S. M. F., 1994, tous droits réservés.

L'accès aux archives de la revue «Bulletin de la S. M. F. » (http: //smf.emath.fr/Publications/Bulletin/Presentation.html) implique l'accord avec les conditions générales d'utilisation (http://www.numdam.org/ conditions). Toute utilisation commerciale ou impression systématique est constitutive d'une infraction pénale. Toute copie ou impression de ce fichier doit contenir la présente mention de copyright.

\section{Numdam}




\title{
ORDRE DE BRUHAT ET TRANSFERT : LE CAS RÉEL
}

\author{
PAR
}

MARTIN ANDLER (*)

RÉSUMÉ. - Soient $G$ un groupe algébrique réductif connexe défini sur $\mathbb{R}$ et $G(\mathbb{R})$ le groupe de ses points réels. On définit un préordre sur l'ensemble des classes d'équivalence de représentations admissibles irréductibles de $G(\mathbb{R})$ et on démontre que ce préordre est conservé par transfert vers un groupe quasi-déployé. Ceci implique des résultats de divisibilité entre fonctions $L$.

ABSTRACT. - Let $G$ be an algebraic reductive connected group defined over $\mathbb{R}$, and $G(\mathbb{R})$ the set of its real points. We define a preorder on the set of equivalence classes of admissible irreducible representations of $G(\mathbb{R})$, and we prove that this preorder is preserved by transfer to a quasi-split group. This implies results of divisibility between $L$-functions.

\section{Introduction}

Considérons un groupe algébrique réductif connexe $G$ défini sur $\mathbb{R}$ et $G(\mathbb{R})$ le groupe de ses points réels. On identifie systématiquement $G$ avec le groupe de ses points complexes et on notera $\mathfrak{g}_{0}$ l'algèbre de Lie de $G(\mathbb{R})$, $\mathfrak{g}$ l'algèbre de Lie complexifiée.

La classification de Langlands établit une correspondance entre certains sous-ensembles finis (les $L$-paquets de représentations) de l'ensemble $\Pi(G(\mathbb{R}))$ des classes de représentations admissibles irréductibles (i.e. les $(\mathfrak{g}, K)$-modules irréductibles admissibles) et les classes de conjugaison (pour l'action de la composante connexe ${ }^{\vee} G$ du $L$-groupe ) dans l'ensemble des homomorphismes quasi-admissibles du groupe de Weil réel $W_{\mathbb{R}}$ dans le $L$-groupe ${ }^{\vee} G^{\Gamma}$ associé à $G(\mathbb{R})([\mathrm{L}],[\mathrm{AV} 1],[\mathrm{ABV}])$; en suivant $[\mathrm{ABV}]$, pour mieux respecter la symétrie entre groupe et $L$-groupe , on note ${ }^{\vee} G$ et ${ }^{\vee} G^{\Gamma}$ au lieu de la notation classique ${ }^{L} G^{0}$ et ${ }^{L} G$ ). On note

(*) Texte reçu le 23 juillet 1992, révisé le 23 février 1993.

M. ANDler, Département de Mathématiques et Informatique (UA 762 du CNRS), École Normale Supérieure, 45 rue d'Ulm, 75230 Paris CEdEx 05.

Classification AMS : $22 \mathrm{E}, 11 \mathrm{~F}$. 
$\mathcal{P}\left({ }^{\vee} G^{\Gamma}\right)$ l'ensemble des homomorphismes quasi-admissibles et $\Phi(G / \mathbb{R})$ le quotient $\mathcal{P}\left({ }^{\vee} G^{\Gamma}\right) /{ }^{\vee} G$.

Soit $\phi$ dans $\Phi(G / \mathbb{R})$. On lui associe un $L$-paquet (fini) de représentations dites standard (séries principales généralisées)

$$
\Psi_{\phi}=\left\{\tau_{1}(\phi), \tau_{2}(\phi), \ldots, \tau_{\ell}(\phi)\right\}
$$

puis pour chacune de ces représentations standard $\tau \in \Psi_{\phi}$ un ensemble fini de sous-quotients (les sous-quotients de Langlands)

$$
\Pi_{\phi}^{\tau}=\left\{\pi_{1}^{\tau}, \ldots, \pi_{k}^{\tau}\right\}
$$

Par définition, le $L$-paquet $\Pi_{\phi}$ est la réunion $\bigcup_{\tau \in \Psi_{\phi}} \Pi_{\phi}^{\tau}$. Cette construction ne dépend que de l'orbite de $\phi$ sous ${ }^{\vee} G$. Il est possible que le $L$-paquet soit vide; la section $\phi$ est dite pertinente si le $L$-paquet est non-vide.

On peut alors définir un préordre sur $\mathcal{P}\left({ }^{\vee} G^{\Gamma}\right) /{ }^{\vee} G$, le L-préordre de Bruhat, de la manière suivante :

$$
\left\{\begin{array}{l}
\phi \prec \phi^{\prime} \text { s'il existe } \tau \in \Psi_{\phi} \text { et } \pi^{\prime} \in \Pi_{\phi^{\prime}} \\
\text { tels que } \pi^{\prime} \text { est un sous-quotient de } \tau \text {. }
\end{array}\right.
$$

On peut formuler la définition autrement, en remplaçant $\Phi(G \backslash \mathbb{R})$ par l'ensemble ${ }^{L} \Pi(G(\mathbb{R}))$ des $L$-paquets de représentations de $G(\mathbb{R})$, ou encore mutatis mutandis, par $\Pi(G(\mathbb{R}))$.

\section{Remarques}

1. - Dans le cas d'un groupe complexe, les $L$-paquets sont réduits à un élément. Le $L$-préordre de Bruhat est une relation d'ordre, qui est isomorphe à l'ordre de Bruhat usuel sur un ensemble de doubles classes d'un groupe de Weyl approprié (voir [D], [A2])

2. - En général, le $L$-préordre de Bruhat n'est pas une relation transitive (voir [V2]).

3. - La relation d'équivalence engendrée par le $L$-préordre de Bruhat est la bloc-équivalence (voir [V1]). On appelle bloc une classe d'équivalence pour la bloc-équivalence. Il est clair que des représentations bloc-équivalentes ont même caractère infinitésimal. La réciproque n'est pas vraie : dans le cas complexe par exemple, les représentations avec caractère infinitésimal donné correspondent à des orbites dans le dual de l'algèbre de Cartan sous le groupe de Weyl. Alors que les blocs correspondent aux orbites sous le groupe de Weyl intégral (voir [A1], [A2]).

$$
\text { TOME } 122-1994-\mathrm{N}^{\circ} 1
$$


Considérons maintenant deux groupes réels $G(\mathbb{R})$ et $G^{\prime}(\mathbb{R})$ et supposons qu'il existe un $L$-homomorphisme $r$ du $L$-groupe ${ }^{\vee} G$ dans le $L$-groupe ${ }^{\vee} G{ }^{\prime}$. L'homomorphisme $r$ induit une application $\mathcal{P}(r) \operatorname{de} \mathcal{P}\left({ }^{\vee} G^{\Gamma}\right)$ dans $\mathcal{P}\left({ }^{\vee} G^{\prime \Gamma}\right)$, qui passe aux quotients de $\mathcal{P}\left({ }^{\vee} G^{\Gamma}\right) /{ }^{\vee} G$ dans $\mathcal{P}\left({ }^{\vee} G^{\prime \Gamma}\right) /{ }^{\vee} G^{\prime}$, et donc une application de ${ }^{L} \Pi\left(G(\mathbb{R})\right.$ ) dans ${ }^{L} \Pi\left(G^{\prime}(\mathbb{R})\right.$ ) appelée transfert (ou parfois lifting). Nous démontrons le théorème suivant.

ThÉORÈme 1. - Supposons $G^{\prime}$ quasi-déployé. L'application $\mathcal{P}(r)$ est croissante pour les L-préordres de Bruhat dans $\mathcal{P}\left({ }^{\vee} G^{\Gamma}\right) /{ }^{\vee} G$ et $\mathcal{P}\left({ }^{\vee} G^{\prime \Gamma}\right) /{ }^{\vee} G^{\prime}$.

Remarque. - La démonstration repose sur une description géométrique de la classification de Langlands contenue dans [ABV]. Cette description est expliquée au paragraphe 2. La restriction sur $G^{\prime}$ est importante : la caractérisation du $L$-préordre de Bruhat dans [ABV] n'en est qu'une condition nécessaire dans le cas non quasi-déployé. En ce qui concerne l'utilisation que nous avons en vue (THÉorÈme 2 infra), ce cas est suffisant. Le cas particulier des groupes complexes avait été démontré dans [A2].

Regardons maintenant les rapports entre préordre de Bruhat et fonctions $L$. Par définition, la fonction $L$ de Langlands (qui ne dépend que du $L$-paquet contenant $\pi) L\left(\pi^{G}, r, s\right)=L(\phi, r, s)$ associée à la représentation $\pi^{G}$ de $G$ et à la représentation rationnelle $r$ est la fonction $L$ d'Artin associée à la représentation $r \circ \phi$ du groupe de Weil $W_{\mathbb{R}}$ (voir le paragraphe 3 ). On a alors :

THÉORÈme 2.- Soit $G$ un groupe réductif réel connexe algébrique, $r$ une représentation rationnelle de son $L$-groupe, et $\pi_{1}, \pi_{2}$ deux représentations irréductibles de $G$ telles que $\pi_{1} \prec \pi_{2}$ pour l'ordre de Bruhat.

(i) Alors le quotient

$$
\frac{L\left(\pi_{2}, r, s\right)}{L\left(\pi_{1}, r, s\right)}
$$

est une fonction entière.

(ii) De plus, si $\pi^{\vee}$ dénote la contraposée de $\pi$, on a

$$
\frac{L\left(\pi_{1}, r, s\right)}{L\left(\pi_{1}^{\vee}, r, 1-s\right)}=* \frac{L\left(\pi_{2}, r, s\right)}{L\left(\pi_{2}^{\vee}, r, 1-s\right)}
$$

où * désigne une fonction entière dont l'inverse est entière. 
REMARQUe. - Les quotients

$$
\frac{L\left(\pi_{1}, r, s\right)}{L\left(\pi_{1}^{\vee}, r, 1-s\right)}
$$

qui interviennent dans l'assertion $(\dagger \dagger)$ du théorème sont, si l'on a pris les bonnes normalisations, à une constante près, les facteurs $\gamma$ de $[\mathrm{J}]$. Le THÉORÈME 2 ne «voyant» pas les constantes, il n'y a pas d'inconvénient à définir ici

$$
\gamma(\pi, r, s)=\frac{L(\pi, r, s)}{L\left(\pi^{\vee}, r, 1-s\right)}
$$

et à reformuler l'assertion (ii) du théorème en :

(ii) ${ }^{\prime}$ De plus,

$$
\gamma\left(\pi_{1}, r, s\right)=* \gamma\left(\pi_{2}, r, s\right)
$$

où * est une fonction entière dont l'inverse est entière.

Dans le cas des groupes complexes, j'avais démontré le THÉORÈmE 2 par des méthodes purement combinatoires dans [A1], puis de manière analogue à ce qui est proposé ici dans [A2].

La démonstration du THÉorÈme 2 se fait en deux étapes : d'abord démontrer le cas particulier des fonctions $L$ principales pour le groupe linéaire; ensuite s'y ramener grâce au THÉoRÈme 1. Le cas particulier résulte immédiatement des résultats de Jacquet sur les fonctions $L$ principales pour les groupes linéaires $[\mathrm{J}]$. Nous en indiquons une autre démonstration combinatoire au paragraphe 4.

Le paragraphe 4 contient également plusieurs calculs d'exemples qui témoignant de la difficulté combinatoire qui est en jeu dans ces questions. Ces calculs, qui paraissent miraculeux, soulignent l'étroitesse du lien entre fonctions $L$ et représentations des groupes réductifs.

A cet égard, il faut signaler que les résultats de divisibilité entre fonctions $L$ sont également vrais dans le cas $p$-adique pour les représentations avec un vecteur fixe sous le groupe d'Iwahori. Cela avait été d'abord démontré par Rodier $[R]$; c'est maintenant une conséquence facile de la classification de Langlands par KAZHDAN-LuszTig dans ce cas (cf. [BM]).

On peut se demander si nos résultats admettent une réciproque. Trivialement, la divisibilité des fonctions $L$ pour toute représentation $r$ n'implique pas le $L$-préordre de Bruhat - car précisément, ce préordre n'est pas un ordre, alors que la relation de divisibilité est toujours transitive.

TOME $122-1994-\mathrm{N}^{\circ} 1$ 
Dans le cas complexe, où le préordre est un ordre, j'avais formulé la conjecture suivante dans [A1] :

1. - Les facteurs $\gamma$ caractérisent le bloc, c'est-à-dire : soient $\pi_{1}$ et $\pi_{2}$ deux représentations telles que, pour tout $r$, on ait

$$
\gamma\left(\pi_{1}, r, s\right)=\gamma\left(\pi_{2}, r, s\right)
$$

Alors $\pi_{1}$ et $\pi_{2}$ sont bloc-équivalentes. ${ }^{1}$

2. - Soient $\pi_{1}$ et $\pi_{2}$ deux représentations bloc-équivalentes; alors la relation de divisibilité implique l'ordre de Bruhat, c'est-à-dire : si pour tout $r$

$$
\frac{L\left(\pi_{2}, r, s\right)}{L\left(\pi_{1}, r, s\right)}
$$

est une fonction entière, alors $\pi_{1} \prec \pi_{2}$ pour l'ordre de Bruhat.

Je remercie Laurent Clozel, Robert P. Langlands et David Vogan pour d'utiles conversations.

\section{Paramètres de Langlands géométriques}

Les définitions de ce paragraphe sont issues de [AV2], [ABV]. On pourra également se référer à [AV1]. Soit $G$ un groupe réductif algébrique défini sur $\mathbb{R}$. Sans risque de confusion, on peut noter aussi $G$ le groupe de ses points complexes. On note $\mathfrak{g}$ l'algèbre de Lie (complexe) de $G$. On a une action $\gamma$ (antiholomorphe) du groupe de Galois $\Gamma$ de $\mathbb{C}$ par rapport à $\mathbb{R}$ sur $G$; indifféremment on notera aussi $\gamma$ l'image par $\gamma$ de l'élément non trivial de $\Gamma$. Le groupe des points réels $G(\mathbb{R})$ est l'ensemble des points fixes de $G$ sous $\gamma$. Fixons une fois pour toutes un tore maximal $T$ et un sous-groupe de Borel $B$. On définit alors la donnée radicielle valuée $\Psi_{0}(G)$ associée à $(G, B, T)$, et on a la suite exacte

$$
1 \rightarrow \operatorname{Int}(G) \longrightarrow \operatorname{Aut}(G) \longrightarrow \operatorname{Aut}\left(\Psi_{0}(G)\right) \rightarrow 1
$$

où $\operatorname{Int}(G), \operatorname{Aut}(G), \operatorname{Aut}\left(\Psi_{0}(G)\right)$ désignent respectivement les automorphismes intérieurs de $G$, les automorphismes de $G$ et les automorphismes de $\Psi_{0}(G)$. En composant $\gamma$ avec l'automorphisme anti-holomorphe correspondant à la forme réelle déployée définie par le choix d'une base de Chevalley, on obtient un élément de $\operatorname{Aut}(G)$, puis un élément $\zeta_{\gamma}$

\footnotetext{
${ }^{1}$ La conjecture dans [A1] est que le facteur $\gamma$ caractérise le caractère infinitésimal. Il est clair que cela est faux et que c'est bien le bloc qui doit être caractérisé par les $\gamma$.
} 
de $\operatorname{Aut}\left(\Psi_{0}(G)\right)$. Cette application établit une bijection entre l'ensemble des classes intérieures de formes réelles de $G$ et l'ensemble des involutions dans $\operatorname{Aut}\left(\Psi_{0}(G)\right)$. Par définition, le $L$-groupe complexe ${ }^{\vee} G$ a pour donnée radicielle la donnée duale de $\Psi_{0}(G)$. Il vient muni d'un tore maximal ${ }^{\vee} T$ et d'un sous-groupe de Borel ${ }^{\vee} B$. On sait que les ensembles d'automorphismes extérieurs de $\Psi_{0}(G)$ et $\Psi_{0}\left({ }^{\vee} G\right)$ sont canoniquement isomorphes. On déduit donc de $\zeta$ un automorphisme ${ }^{\vee} \zeta$ de $\Psi_{0}\left({ }^{\vee} G\right)$. Par définition, ${ }^{\vee} \zeta$ ne dépend que de la classe intérieure de la forme réelle $G(\mathbb{R})$.

La demi-somme $\rho$ des racine positives correspondant au choix de $B$ est un élément du dual de l'algèbre de Lie $\mathfrak{t}$, et donc un élément de ${ }^{\vee} \mathfrak{t}$. Soit ${ }^{\vee} z(\rho)=\exp (2 i \pi \rho)$; c'est un élément du centre de ${ }^{\vee} G$.

On définit alors le $L$-groupe associé à la classe intérieure de $G(\mathbb{R})$ comme le couple formé d'un groupe algébrique complexe ${ }^{\vee} G^{\Gamma}$, contenant ${ }^{\vee} G$ comme sous-groupe d'indice 2 , et d'une classe de conjugaison ${ }^{\vee} \mathcal{D}$ de couples $(\delta, B)$, où ${ }^{\vee} \delta$ est un élément d'ordre fini dans ${ }^{\vee} G^{\Gamma} \backslash \backslash^{\vee} G,{ }^{\vee} B$ est un sous groupe de Borel de ${ }^{\vee} G$, et les propriétés suivantes sont vérifiées :

(1) Soit ${ }^{\vee} \theta$ l'application de ${ }^{\vee} G$ dans lui-même :

$$
x \longmapsto \delta x \delta^{-1} ;
$$

${ }^{\vee} \theta$ est une involution principale, i.e. il existe un élément semi-simple régulier ${ }^{\vee} X$ de ${ }^{\vee} \mathfrak{g}$ tel que ${ }^{\vee} \theta\left({ }^{\vee} X\right)=-{ }^{\vee} X$.

(2) ${ }^{\vee} \theta\left({ }^{\vee} B\right)={ }^{\vee} B$.

(3) ${ }^{\vee} \delta^{2}={ }^{\vee} z(\rho)$.

(4) Les racines simples (par rapport au choix de $^{\vee} B$ ) sont complexes $\left({ }^{\vee} \theta\left({ }^{\vee} \alpha\right) \neq \pm \alpha\right)$ ou imaginaires non compactes $\left({ }^{\vee} \theta(\alpha)=\alpha\right.$ et le vecteur radiciel correspondant vérifie $\left.{ }^{\vee} \theta\left({ }^{\vee} X \vee_{\alpha}\right)=-{ }^{\vee} X \vee_{\alpha}\right)$.

La condition (1) signifie que ${ }^{\vee} \theta$ est l'involution de Cartan d'une forme réelle quasi-déployée de $G$.

On considère le groupe de Weil $W_{\mathbb{R}}$, extension non triviale du groupe de Weil complexe $W_{\mathbb{C}}=\mathbb{C}^{\times}$par $\Gamma$. Le groupe est donc engendré par $\mathbb{C}^{\times}$ et un élément $j$ avec les relations $j^{2}=-1$ et $j z j^{-1}=\bar{z}$ pour $z$ in $\mathbb{C}^{\times}$. On identifie $W_{\mathbb{R}}$ au produit $\mathbb{C}^{\times} \times\{-1,1\}$ avec la loi :

$$
(z, \epsilon)\left(z^{\prime}, \epsilon^{\prime}\right)=\left(z \epsilon\left(z^{\prime}\right) \alpha\left(\epsilon, \epsilon^{\prime}\right), \epsilon \epsilon^{\prime}\right)
$$

où $\quad \alpha\left(\epsilon, \epsilon^{\prime}\right)=\left\{\begin{array}{rl}1 & \text { si } \epsilon \text { ou } \epsilon^{\prime}=1, \\ -1 & \text { si } \epsilon \text { et } \epsilon^{\prime}=1,\end{array} \quad \epsilon\left(z^{\prime}\right)= \begin{cases}z^{\prime} & \text { si } \epsilon=1, \\ \bar{z}^{\prime} & \text { si } \epsilon=-1 .\end{cases}\right.$

TOME $122-1994-\mathrm{N}^{\circ} 1$ 
On a la suite exacte

$$
1 \rightarrow W_{\mathbb{C}} \longrightarrow W_{\mathbb{R}} \longrightarrow \Gamma \rightarrow 1,
$$

où la flèche $W_{\mathbb{R}} \rightarrow \Gamma$ est donnée par $(z, \epsilon) \mapsto \epsilon$. On a une autre suite exacte

$$
1 \rightarrow \mathbb{U} \longrightarrow W_{\mathbb{R}} \longrightarrow \mathbb{R} \rightarrow 1
$$

où $\mathbb{U}$ est l'ensemble des complexes de module 1 , et la flèche $W_{\mathbb{R}} \rightarrow \mathbb{R}^{\times}$ est donnée par $(z, \epsilon) \mapsto \epsilon|z|$, avec, suivant l'usage en théorie des nombres, $|z|=z \bar{z}$.

Un homomorphisme quasi-admissible est par définition un homomorphisme continu $\phi$ de $W_{\mathbb{R}}$ dans le $L$-groupe ${ }^{\vee} G^{\Gamma}$ tel que l'image de $W_{\mathbb{C}}$ est formée d'éléments semi-simples et tel que le diagramme suivant soit commutatif :

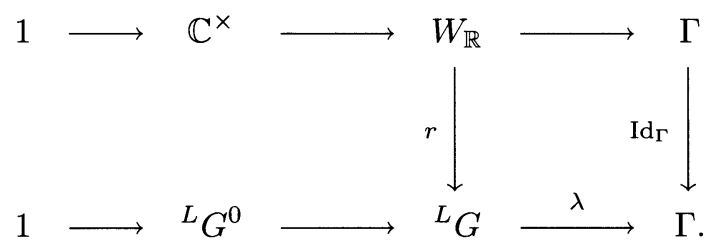

Notons $\mathcal{P}\left({ }^{\vee} G^{\Gamma}\right)$ l'ensemble des homomorphismes quasi-admissibles et $\Phi(G / \mathbb{R})$ l'ensemble des classes de conjugaison de $\mathcal{P}\left({ }^{\vee} G^{\Gamma}\right)$ sous l'action de ${ }^{\vee} G$ (puisque le $L$-groupe ne dépend que de la classe intérieure de la forme réelle, les ensembles $\mathcal{P}\left({ }^{\vee} G^{\Gamma}\right)$ et $\Phi(G / \mathbb{R})$ ne dépendent que de la classe intérieure de $G(\mathbb{R})$ ).

La paramétrisation de Langlands associe à tout élément de $\Phi(G / \mathbb{R})$ un $L$-paquet de représentations irréductibles et un $L$-paquet de représentations standard (éventuellement vides si l'homomorphisme n'est pas pertinent).

Il n'est pas difficile $\left[\mathrm{ABV}\right.$, section 4] de voir que les éléments de $\mathcal{P}\left({ }^{\vee} G^{\Gamma}\right)$ sont en bijection avec l'ensemble des couples $(y, \lambda)$ qui vérifient :

(i) $y \in\left({ }^{\vee} G^{\Gamma} \backslash{ }^{\vee} G\right)$ et $\lambda$ semi-simple dans ${ }^{\vee} \mathfrak{g}$;

(ii) $y^{2}=\exp (2 i \pi \lambda)$;

(iii) $[\lambda, \operatorname{Ad} y(\lambda)]=0$, l'élément $\phi$ associé à $(y, \lambda)$ étant donné par :

$$
\begin{aligned}
& \phi(z)=z^{\lambda} \bar{z}^{\operatorname{Ad} y(\lambda)} \quad \text { pour } \quad z \in b C^{\times}, \\
& \phi(j)=\exp (-\pi i \lambda) y .
\end{aligned}
$$

BULLETIN DE LA SOCIÉTÉ MATHÉMATIQUE DE FRANCE 
Pour un élément semi-simple donné $\lambda$ de ${ }^{\vee} \mathfrak{g}$, on définit

$$
\begin{aligned}
& { }^{\vee} \mathfrak{g}(\lambda)_{n}=\left\{\mu \in \in^{\vee} \mathfrak{g} ;[\lambda, \mu]=n \mu\right\}, \\
& \vee \mathfrak{g}(\lambda)=\sum_{n \in \mathbb{Z}} v^{\vee} \mathfrak{g}(\lambda)_{n}, \\
& \vee_{\mathfrak{l}}(\lambda)={ }^{\vee} \mathfrak{g}(\lambda)_{0}, \\
& v_{\mathfrak{n}}(\lambda)=\sum_{n \in \mathbb{N}^{*+}} v_{\mathfrak{g}}(\lambda)_{n}, \\
& \vee_{\mathfrak{p}}(\lambda)=v^{\vee}(\lambda)+v^{\vee} \mathfrak{n}(\lambda),
\end{aligned}
$$

puis

$$
e(\lambda)=\exp (2 i \pi \lambda)
$$

enfin

(11) ${ }^{\vee} G(\lambda)=$ le centralisateur dans ${ }^{\vee} G$ de $e(\lambda)$,

(12) ${ }^{\vee} L(\lambda)=$ le centralisateur dans ${ }^{\vee} G$ de $\lambda$,

(13) ${ }^{\vee} N(\lambda)=$ sous-groupe unipotent connexe d'algèbre de Lie ${ }^{\vee} \mathfrak{n}(\lambda)$,

(14) ${ }^{\vee} P(\lambda)={ }^{\vee} L(\lambda)^{\vee} N(\lambda)$.

Le plateau canonique (en anglais, canonical flat) $\mathcal{F}(\lambda)$ associé à $\lambda$ est par définition l'espace affine $\lambda+{ }^{\vee} \mathfrak{n}(\lambda)$. On a (lemme 6.3 de [ABV]) :

LeMme 1.

(1) ${ }^{\vee} G(\lambda)$ est un sous-groupe réductif connexe d'algèbre de Lie ${ }^{\vee} \mathfrak{g}(\lambda)$;

(2) ${ }^{\vee} L(\lambda)$ est un sous-groupe réductif connexe d'algèbre de Lie ${ }^{\vee} \mathfrak{l}(\lambda)$;

(3) ${ }^{\vee} N(\lambda)$ est un sous-groupe connexe de ${ }^{\vee} G(\lambda)$;

(4) ${ }^{\vee} P(\lambda)$ est un sous-groupe parabolique de ${ }^{\vee} G(\lambda)$ d'algèbre de Lie $\vee_{\mathfrak{p}}(\lambda)$ et de décomposition de Levi ${ }^{\vee} P(\lambda)={ }^{\vee} L(\lambda)^{\vee} N(\lambda)$;

(5) $\operatorname{Ad}(P(\lambda)) \cdot \lambda=\lambda+{ }^{\vee} \mathfrak{n}(\lambda)=\mathcal{F}(\lambda)$ et l'application ${ }^{\vee} n \mapsto{ }^{\vee} n \cdot \lambda$ est un isomorphisme de variétés algébriques.

On déduit de ce lemme que $e(\lambda),{ }^{\vee} G(\lambda),{ }^{\vee} N(\lambda),{ }^{\vee} P(\lambda)$ ne dépendent que du plateau canonique $\mathcal{F}=\mathcal{F}(\lambda)$ et que le stabilisateur de $\mathcal{F}(\lambda)$ dans ${ }^{\vee} G$ est $P(\lambda)$. On note respectivement $e(\mathcal{F}),{ }^{\vee} G(\mathcal{F}),{ }^{\vee} N(\mathcal{F})$ et ${ }^{\vee} P(\mathcal{F})$ pour $e(\lambda),{ }^{\vee} G(\lambda),{ }^{\vee} N(\lambda)$ et ${ }^{\vee} P(\lambda)$.

$$
\text { TOME } 122-1994-\mathrm{N}^{\circ} 1
$$


Considérons maintenant l'ensemble $\mathcal{X}\left({ }^{\vee} G^{\Gamma}\right)$ des paramètres géométriques

$\mathcal{X}\left({ }^{\vee} G^{\Gamma}\right)=\left\{(y, \mathcal{F}) \mid y \in{ }^{\vee} G^{\Gamma} \backslash^{\vee} G, \mathcal{F}\right.$ un plateau canonique, $\left.y^{2}=e(\mathcal{F})\right\}$.

Cet ensemble est muni d'une action de $^{\vee} G$. On a une application de $\mathcal{P}\left({ }^{\vee} G^{\Gamma}\right)$ dans $\mathcal{X}\left({ }^{\vee} G^{\Gamma}\right)$ définie par :

$$
(y, \lambda) \longmapsto(y, \mathcal{F}(\lambda)) .
$$

Cette application est clairement ${ }^{\vee} G$-équivariante. D'après la proposition 6.17 de [ABV], elle induit une bijection au niveau des quotients :

$$
\mathcal{P}\left({ }^{\vee} G^{\Gamma}\right) /{ }^{\vee} G \stackrel{\sim}{\longrightarrow} \mathcal{X}\left({ }^{\vee} G^{\Gamma}\right) /{ }^{\vee} G .
$$

On peut donc prendre le quotient $\mathcal{X}\left({ }^{\vee} G^{\Gamma}\right) /{ }^{\vee} G \simeq \mathcal{X}\left({ }^{\vee} G^{\Gamma}\right) /{ }^{\vee} G=\Phi(G, \mathbb{R})$ comme ensemble de paramètres pour la classification de Langlands.

Munissons cet ensemble d'une topologie. Soit $\mathcal{O}$ une ${ }^{\vee} G$-orbite semisimple dans $\mathfrak{v} \mathfrak{g}$. On définit :

$$
\begin{aligned}
\mathcal{X}\left(\mathcal{O},{ }^{\vee} G^{\Gamma}\right) & =\left\{(y, \mathcal{F}) \in \mathcal{X}\left({ }^{\vee} G^{\Gamma}\right), \mathcal{F} \subset \mathcal{O}\right\}, \\
\mathcal{P}(\mathcal{O}) & =\text { l'ensemble des plateaux canoniques dans } \mathcal{O}, \\
\mathcal{C}(\mathcal{O}) & =\left\{g e(\mathcal{F}) g^{-1} \mid g \in{ }^{\vee} G\right\}, \\
\mathcal{I}(\mathcal{O}) & =\left\{y \in{ }^{\vee} G^{\Gamma} \backslash{ }^{\vee} G \mid y^{2} \in \mathcal{C}(\mathcal{O})\right\} .
\end{aligned}
$$

Choisissons $\mathcal{F}$ inclus dans $\mathcal{O}$. Comme le stabilisateur de $\mathcal{F}$ est $P(\mathcal{F})$, on a $\mathcal{P}(\mathcal{O}) \simeq{ }^{\vee} G / P(\mathcal{F})$. D'autre part, clairement $\mathcal{C}(\mathcal{O}) \simeq{ }^{\vee} G /{ }^{\vee} G(\mathcal{F})$. Cela prouve que $\mathcal{P}(\mathcal{O})$ et $\mathcal{C}(\mathcal{O})$ sont des variétés algébriques. L'application $\mathcal{P}(\mathcal{O}) \rightarrow \mathcal{C}(\mathcal{O}): \mathcal{F} \mapsto e(\mathcal{F})$ coïncide, modulo les identifications précédentes, avec l'application canonique ${ }^{\vee} G / P(\mathcal{F}) \rightarrow{ }^{\vee} G /{ }^{\vee} G(\mathcal{F})$ et est donc algébrique. D'autre part $\mathcal{I}(\mathcal{O})$ est algébrique, et on démontre [ABV, prop. 6.13] que les orbites de ${ }^{\vee} G$ dans $\mathcal{I}(\mathcal{O})$ sont en nombre fini et toutes fermées. On a l'isomorphisme ${ }^{\vee} G$ équivariant $\mathcal{X}\left(\mathcal{O},{ }^{\vee} G^{\Gamma}\right) \simeq$ $\mathcal{P}(\mathcal{O}) \times_{\mathcal{C}(\mathcal{O})} \mathcal{I}(\mathcal{O})$ (où $\times_{\mathcal{C}(\mathcal{O})}$ désigne le produit fibré au dessus de $\mathcal{C}(\mathcal{O})$, et l'action de ${ }^{\vee} G$ sur le produit fibré est l'action par conjugaison sur les deux facteurs) - ce qui donne à $\mathcal{X}\left(\mathcal{O},{ }^{\vee} G^{\Gamma}\right)$ la structure d'une variété algébrique. On montre que ${ }^{\vee} G$ y a un nombre fini d'orbites.

En fin de compte, on munit $\mathcal{X}\left({ }^{\vee} G^{\Gamma}\right)$ de la topologie où les $\mathcal{X}\left(\mathcal{O},{ }^{\vee} G^{\Gamma}\right)$ sont ouverts et fermés, et où la topologie sur chaque $\mathcal{X}\left(\mathcal{O},{ }^{\vee} G^{\Gamma}\right)$ est sa topologie de variété algébrique. 
L-préordre de Bruhat et paramètres géométriques. - Soit $\Pi(G(\mathbb{R}))$ l'ensemble des $(\mathfrak{g}, K)$-modules admissibles irréductibles de $G(\mathbb{R})$. La reformulation de la paramétrisation de Langlands en termes de ${ }^{\vee} G$-orbites dans $\mathcal{X}\left({ }^{\vee} G^{\Gamma}\right)$ nous permet d'utiliser les notations suivantes : à une ${ }^{\vee} G$-orbite $\mathcal{S}$ dans $\mathcal{X}\left({ }^{\vee} G^{\Gamma}\right)$ on associe le $L$-paquet de représentations standard $\Psi_{S}$, pour chaque $\tau$ dans $\Psi_{S}$ l'ensemble (fini) de ses sous-quotients de Langlands irréductibles $\Pi_{S}^{\tau}$, et le $L$-paquet de représentations irréductibles $\Pi_{S}=\bigcup_{\tau \in \Psi_{S}} \Pi_{S}^{\tau}$.

On a défini dans l'introduction le $L$-préordre de Bruhat, qu'on peut décrire explicitement par le résultat suivant [ABV, prop. 1.11].

Proposition. - Soit $\pi$ et $\pi^{\prime}$ dans $\Pi(G(\mathbb{R}))$, respectivement dans les L-paquets $\Pi_{S}$ et $\Pi_{S^{\prime}}$, et supposons $\pi \prec \pi^{\prime}$ pour le L-préordre de Bruhat. Alors $S \subset \bar{S}^{\prime}$. La réciproque est vraie si le groupe $G(\mathbb{R})$ est quasi-déployé.

\section{Fonctions $L$}

Dans ce paragraphe, nous rappelons les définitions concernant les fonctions $L$ locales. Les références sont $[\mathrm{Ta}]$ et [Bo]. Les représentations irréductibles de $W_{\mathbb{R}}$ sont bien connues. Elles sont de deux types :

- Les caractères $\chi_{\lambda, \alpha}$, avec $\lambda \in \mathbb{C}$ et $\alpha=0$ ou 1 , qui proviennent (voir la suite exacte $(3))$ des caractères de $\mathbb{R}^{\times}$

$$
\chi(x)=\left(\frac{x}{|x|}\right)^{\alpha} x^{\lambda}
$$

et sont donc donnés par

$$
(z, \epsilon) \longmapsto \epsilon^{\alpha}|z|^{\lambda}
$$

- Les représentations $\theta_{p, q}$, où $p, q$ sont complexes avec $p-q \in \mathbb{Z} \backslash 0$, induites à partir des caractères $\xi_{p, q}$ de $\mathbb{C}^{\times}$(voir la suite exacte $(2)$ ) :

$$
\xi_{p, q}(z)=z^{p} \bar{z}^{q}
$$

$\theta_{p, q}$ est de dimension 2 , donnée par

$$
\begin{aligned}
& z \mapsto\left(\begin{array}{cc}
z^{p} \bar{z}^{q} & 0 \\
0 & \bar{z}^{p} z^{q}
\end{array}\right), \\
& j \mapsto\left(\begin{array}{cc}
0 & (-1)^{p-q} \\
1 & 0
\end{array}\right) .
\end{aligned}
$$

On remarque que $\theta_{p, q}$ devient réductible quand $p=q$; on vérifie que $\theta_{p, p}$ est équivalente à la somme $\chi_{p, 0}+\chi_{p, 1}$.

$$
\text { TOME } 122-1994-\mathrm{N}^{\circ} 1
$$


Les fonctions $L$

- La fonction $L$ associée à $\chi_{\lambda, \alpha}$ est :

$$
L(\chi, s)=\pi^{-\frac{1}{2}(\lambda+\alpha+s)} \Gamma\left(\frac{1}{2}(\lambda+\alpha+s)\right) .
$$

- La fonction $L$ associée à $\theta_{p, q}$ est :

$$
L(\xi, s)=2(2 \pi)^{-(\max (p, q)+s)} \Gamma(\max (p, q)+s) .
$$

On remarque que dans le cas où $\theta_{p, q}$ est réductible, c'est-à-dire dans le cas $p=q$, on a :

$$
L\left(\theta_{p, p}, s\right)=L\left(\chi_{p, 0}, s\right) L\left(\chi_{p, 1}, s\right)
$$

En effet, on a :

$$
\begin{aligned}
L\left(\theta_{p, p}, s\right)= & 2(2 \pi)^{-(p+s)} \Gamma(p+s) \\
= & 2(2 \pi)^{-(p+s)} \pi^{-\frac{1}{2}} 2^{s-1} \Gamma\left(\frac{1}{2}(p+s)\right) \Gamma\left(\frac{1}{2}(p+s+1)\right) \\
& \quad(\text { par duplication de la fonction } \Gamma) \\
= & \pi^{-\frac{1}{2}(p+s)} \Gamma\left(\frac{1}{2}(p+s)\right) \pi^{-\frac{1}{2}(p+s+1)} \Gamma\left(\frac{1}{2}(p+s+1)\right) .
\end{aligned}
$$

Cette remarque permet d'étendre la construction aux représentations de $W_{\mathbb{R}}$, en sorte que la fonction $L$ d'une somme de représentations (au sens du groupe de Grothendieck) est le produit des fonctions $L$.

On peut maintenant définir les fonctions $L$ de Langlands. Soit $\phi$ dans $\mathcal{P}\left({ }^{\vee} G^{\Gamma}\right)$, et $r$ une représentation de ${ }^{\vee} G^{\Gamma}$ (on entend par là une représentation de dimension finie de ${ }^{\vee} G^{\Gamma}$ dont la restriction à ${ }^{\vee} G$ est rationnelle). Alors $r \circ \phi$ est une représentation de dimension finie de $W_{\mathbb{R}}$, à quoi on sait associer une fonction $L$. C'est par définition la fonction $L$ associée à $\phi$ et $r$, ou encore la fonction $L$ associée au $L$-paquet $\Pi_{\phi}$ et à $r$.

Supposons maintenant que $M$ est la partie de Langlands d'un parabolique cuspidal $M A N$, et que la représentation $\pi_{G}$ de $G$ est un sous-quotient de Langlands de la représentation induite de $M A N$ à $G$ de la représentation $\pi_{M} \otimes e^{\nu} \otimes 1$. Alors, il existe un $L$-homomorphisme du $L$-groupe ${ }^{\vee} M^{\Gamma}$ dans ${ }^{\vee} G^{\Gamma}$, qui permet de considérer les représentations de ${ }^{\vee} G^{\Gamma}$ comme des représentations de ${ }^{\vee} M^{\Gamma}$. Par construction, les fonctions $L$ associées à $\pi_{G}$ et $\pi_{M}$ et une représentation $r$ de ${ }^{\vee} G^{\Gamma}$ coïncident. 


\section{Le cas de $\operatorname{GL}(n, \mathbb{R})$}

Dans ce paragraphe, on démontre le ThÉorème 2 dans le cas des fonctions $L$ principales pour $\mathrm{GL}(n, \mathbb{R})$ et on calcule quelques exemples.

Fixons les notations. Le $L$-groupe ${ }^{\vee} G$ de $G=\mathrm{GL}(n, \mathbb{R})$ est le produit direct de $\mathrm{GL}(n, \mathbb{C})$ par le groupe de Galois $\Gamma$. La donnée d'un homomorphisme admissible de $W_{\mathbb{R}}$ dans ${ }^{\vee} G$ est équivalente à celle d'un homomorphisme de $W_{\mathbb{R}}$ dans $\operatorname{GL}(n, \mathbb{C})$, c'est-à-dire une représentation de dimension $n$ de $W_{\mathbb{R}}$, qui est nécessairement complètement réductible, donc somme de caractères et de représentations de dimension 2 .

Nous indiquons deux démonstrations du cas particulier. La première utilise $[\mathrm{J}]$ et nous en donnons maintenant l'argument en utilisant les notations de loc. cit.

Soient $\pi$ une représentation admissible de $G=\mathrm{GL}(n, \mathbb{R}), f$ un coefficient $K$-fini de $\pi$ et $\Psi$ une fonction sur l'ensemble des matrices $n \times n$ à coefficients réels de la forme $\Psi(g)=P\left(g_{i j}\right) \exp \left(-\pi \sum_{i j} g_{i j}^{2}\right)$, où $P$ est un polynôme. Considérons l'intégrale

$$
Z(\Psi, f, s)=\int_{G} \Psi(g)|\operatorname{det} g|^{s} f(g) \mathrm{d} g,
$$

où $\mathrm{d} g$ est une mesure de Haar sur $\mathrm{GL}(n, \mathbb{R})$.

Le paragraphe 4 de $[\mathrm{J}]$ contient les faits suivants :

- Supposons que $\pi$ est irréductible, soit une série principale généralisée. Alors l'intégrale $Z(\Psi, f, s)$ converge dans un demi-plan $\operatorname{Re} s \geq \operatorname{Re} s_{0}$ et se prolonge en une fonction méromorphe.

- Si $\pi$ est soit le sous-quotient de Langlands $\pi_{\phi}$ associé à $\phi \in \mathcal{P}\left({ }^{\vee} G^{\Gamma}\right)$, soit la représentation standard $\tau_{\phi}$ associée à $\phi \in \mathcal{P}\left({ }^{\vee} G^{\Gamma}\right)$, pour tout $\Psi$ et tout coefficient $f$, l'intégrale $Z(\Psi, f, s)$ est un multiple de la fonction $L(\phi, s)$ par un polynôme en $s$ et la fonction $L$ est le pgcd des $Z(\Psi, f, s)$.

- En particulier, si $\pi^{\prime}$ est un autre sous-quotient de $\tau_{\phi}$, associée dans la classification à $\phi^{\prime}$, les coefficients de $\pi^{\prime}$ étant des coefficients de $\tau_{\phi}$, on a

$$
L\left(\phi^{\prime}, s\right)=P(s) L(\phi, s),
$$

où $P$ est un polynôme. Ceci démontre la première partie du cas particulier du ThÉORÈme 1.

- Soit $\Psi^{\wedge}$ la transformée de Fourier de $\Psi$ relativement au caractère $x \mapsto \exp (2 i \pi x), \pi^{\vee}$ la représentation contragrédiente de $\pi$, et $f^{\vee}$ le coefficient de $\pi^{\vee}$ contragrédient de $f$. On a l'équation fonctionnelle

$$
Z\left(\Psi^{\wedge}, f^{\vee}, 1-s+\frac{1}{2}(n-1)\right)=\gamma(\pi, s) Z(\Psi, f, s),
$$

où $\gamma$ est une fonction méromorphe.

$$
\text { TOME } 122-1994-\mathrm{N}^{\circ} 1
$$


- Posons

$$
\epsilon(\pi, s)=\gamma(\pi, s) \frac{L(\phi, s)}{L\left(\phi^{\vee}, 1-s\right)},
$$

où $\pi$ est comme précédemment soit la représentation standard, soit la représentation irréductible associée à $\phi$, et où $\phi^{\vee}$ désigne le paramètre correspondant à la représentation contragrédiente de $\pi ; \epsilon(s, \pi)$ est une constante. Ceci prouve la deuxième assertion du cas particulier considéré.

La deuxième démonstration est combinatoire. On utilise la description de la série de composition des séries principales généralisées pour $\mathrm{GL}(n, \mathbb{R})$ (cf. [S], [SV]). Comme ce problème peut être réduit aux cas $n=2,3,4$, et comme cette réduction est compatible avec les calculs à effectuer sur les fonctions $L$, il suffit de démontrer le résultat pour $n=2,3,4$.

Réduction aux cas de $\mathrm{GL}(2, \mathbb{R}), \mathrm{GL}(3, \mathbb{R}), \mathrm{GL}(4, \mathbb{R})$. - Soient $G=$ $\mathrm{GL}(n, \mathbb{R})$ et $P=M A N$ un sous-groupe parabolique cuspidal. Soient $\pi_{M}$ une représentation de la série discrète de $M$ et $\nu$ un caractère de $A$. On sait que la représentation standard

$$
\pi_{G}\left(P, \pi_{M}, \nu\right)=\operatorname{Ind}_{M A N}^{G} \pi \otimes \nu \otimes 1
$$

est, dans le groupe de Grothendieck, indépendante de $N$. On choisit $N$ telle que $\nu$ soit dominant par rapport à $N$. Soit $\bar{P}$ le parabolique opposé à $P$. L'opérateur d'entrelacement long (voir [La], [SV]) entre $\pi_{G}\left(P, \pi_{M}, \nu\right)$ et $\pi_{G}\left(\bar{P}, \pi_{M}, \nu\right)$ a pour image la somme directe des sous-quotients de Langlands. On le décompose en un produit d'opérateurs d'entrelacement [SV, §3]. Le corollaire 3.15 de [SV] affirme que les sous-quotients de $\pi_{G}\left(\pi_{M}, \nu\right)$, autres que les sous-quotients de Langlands, sont des facteurs de composition du noyau de l'un des facteurs du produit d'opérateurs d'entrelacement. Enfin, chaque facteur du produit est induit à partir d'opérateurs d'entrelacement correspondant à la situation analogue pour des représentations de sous-groupes de Levi induites à partir de sous-groupes paraboliques de rang réel 1. C'est pourquoi l'étude des suites de composition pour les représentations standard de $\mathrm{GL}(n, \mathbb{R})$ se réduit aux cas suivants :

- $n=2, M$ le parabolique minimal;

- $n=3, M A$ le sous-groupe de Levi formé des matrices de la forme

$$
\left(\begin{array}{lll}
* & * & 0 \\
* & * & 0 \\
0 & 0 & *
\end{array}\right)
$$


- $n=4, M A$ le sous-groupe de Levi formé des matrices de la forme

$$
\left(\begin{array}{llll}
* & * & 0 & 0 \\
* & * & 0 & 0 \\
0 & 0 & * & * \\
0 & 0 & * & *
\end{array}\right) .
$$

Le cas de $G=\mathrm{GL}(2, \mathbb{R})$. - Il y a deux types de représentations irréductibles :

- Les représentations de la série discrète $\pi_{[p, q]}$, qui correspondent aux représentations $\theta_{p, q}$ de $W_{\mathbb{R}}$. On a l'équivalence $\pi_{[p, q]} \sim \pi_{[q, p]}$ qui correspond à la conjugaison de $\theta_{p, q}$ et $\theta_{q, p}$ modulo le $L$-groupe ${ }^{\vee} G$. Dans ce cas, la représentation irréductible est égale à la représentation standard.

- Les sous-quotients de Langlands $\pi_{\left(\lambda_{1}, \alpha_{1}, \lambda_{2}, \alpha_{2}\right)}$ des représentations de la série principale $\tau_{\left(\lambda_{1}, \alpha_{1}, \lambda_{2}, \alpha_{2}\right)}$, (unitairement) induites à partir du caractère du parabolique minimal $M A N$

$$
\left(\begin{array}{cc}
x_{1} & * \\
0 & x_{2}
\end{array}\right) \longmapsto\left(\frac{x_{1}}{\left|x_{1}\right|}\right)^{\alpha_{1}}\left|x_{1}\right|^{\lambda_{1}}\left(\frac{x_{2}}{\left|x_{2}\right|}\right)^{\alpha_{2}}\left|x_{2}\right|^{\lambda_{2}} .
$$

Ces représentations correspondent aux représentations de $W_{\mathbb{R}}$ qui sont somme des deux caractères $\chi_{\lambda_{1}, \alpha_{1}}$ et $\chi_{\lambda_{2}, \alpha_{2}}$. On a l'équivalence

$$
\pi_{\left(\lambda_{1}, \alpha_{1}, \lambda_{2}, \alpha_{2}\right)} \sim \pi_{\left(\lambda_{2}, \alpha_{2}, \lambda_{1}, \alpha_{1}\right)},
$$

qui correspond à la conjugaison de $\chi_{\lambda_{1}, \alpha_{1}}+\chi_{\lambda_{2}, \alpha_{2}}$ et de $\chi_{\lambda_{2}, \alpha_{2}}+\chi_{\lambda_{1}, \alpha_{1}}$ modulo le $L$-groupe ${ }^{\vee} G$.

Il y a un seul cas où la représentation standard $\tau_{\left(\lambda_{1}, \alpha_{1}, \lambda_{2}, \alpha_{2}\right)}$ est réductible : lorsque $\left(\lambda_{1}-\lambda_{2}\right)$ est entier non nul de parité $\alpha_{1}+\alpha_{2}+1$. Dans ce cas, la représentation standard a une suite de composition à deux termes. On peut supposer $\lambda_{1}-\lambda_{2}>0$, le sous-quotient de Langlands est un quotient; c'est la représentation de dimension $\left(\lambda_{1}-\lambda_{2}\right)$ de plus haut poids

$$
\left(\begin{array}{ll}
a & 0 \\
0 & b
\end{array}\right) \longmapsto\left(\frac{a}{|a|}\right)^{\alpha_{1}}|a|^{\lambda_{1}-\frac{1}{2}}\left(\frac{a}{|a|}\right)^{\alpha_{2}}|a|^{\lambda_{2}+\frac{1}{2}}
$$

et la série discrète $\pi_{\left[\lambda_{1}, \lambda_{2}\right]}$ est un sous- module.

D'après (16) et (17), la fonction $L$ principale associée à $\pi_{\left(\lambda_{1}, \alpha_{1}, \lambda_{2}, \alpha_{2}\right)}$ (ou à $\chi_{\lambda_{1}, \alpha_{1}}+\chi_{\lambda_{2}, \alpha_{2}}$ ) est

$$
\begin{aligned}
& L\left(\pi_{\left(\lambda_{1}, \alpha_{1}, \lambda_{2}, \alpha_{2}\right)}, s\right)=\pi^{-\frac{1}{2}\left(\lambda_{1}+\alpha_{1}+s\right)} \pi^{-\frac{1}{2}\left(\lambda_{2}+\alpha_{2}+s\right)} \\
& \Gamma\left(\frac{1}{2}\left(\lambda_{1}+\alpha_{1}+s\right)\right) \Gamma\left(\frac{1}{2}\left(\lambda_{2}+\alpha_{2}+s\right)\right)
\end{aligned}
$$

TOME $122-1994-\mathrm{N}^{\circ} 1$ 
et celle associée à $\pi_{[p, q]}\left(\right.$ ou à $\left.\theta_{p, q}\right)$ est :

$$
L\left(\pi_{[p, q]}, s\right)=2(2 \pi)^{-(\max (p, q)+s)} \Gamma(\max (p, q)+s) .
$$

Le théorème de divisibilité des fonctions $L$ revient donc à montrer que, si $\left(\lambda_{1}-\lambda_{2}\right)$ est entier congru à $\alpha_{1}+\alpha_{2}+1$, le quotient

$$
q(s)=\frac{L\left(\pi_{\left[\lambda_{1}, \lambda_{2}\right]}, s\right)}{L\left(\pi_{\left(\lambda_{1}, \alpha_{1}, \lambda_{2}, \alpha_{2}\right)}, s\right)}
$$

est une fonction entière. On a :

$$
q(s)=\frac{2(2 \pi)^{-\left(\max \left(\lambda_{1}, \lambda_{2}\right)+s\right)} \Gamma\left(\max \left(\lambda_{1}, \lambda_{2}\right)+s\right)}{\pi^{-\frac{1}{2}\left(\lambda_{1}+\alpha_{1}+s\right)} \pi^{-\frac{1}{2}\left(\lambda_{2}+\alpha_{2}+s\right)} \Gamma\left(\frac{1}{2}\left(\lambda_{1}+\alpha_{1}+s\right)\right) \Gamma\left(\frac{1}{2}\left(\lambda_{2}+\alpha_{2}+s\right)\right)} .
$$

Il nous est loisible de supposer que $\left(\lambda_{1}-\lambda_{2}\right)$ est un entier positif. L'expression devient

$$
q(s)=\frac{2(2 \pi)^{-\left(\lambda_{1}+s\right)} \Gamma\left(\lambda_{1}+s\right)}{\pi^{-\frac{1}{2}\left(\lambda_{1}+\alpha_{1}+s\right)} \Gamma\left(\frac{1}{2}\left(\lambda_{1}+\alpha_{1}+s\right)\right) \pi^{-\frac{1}{2}\left(\lambda_{2}+\alpha_{2}+s\right)} \Gamma\left(\frac{1}{2}\left(\lambda_{2}+\alpha_{2}+s\right)\right)} .
$$

Appliquons la formule de duplication de la fonction $\Gamma$ :

$$
\Gamma(z)=2^{z-1} \pi^{-\frac{1}{2}} \Gamma\left(\frac{1}{2} z\right) \Gamma\left(\frac{1}{2}(z+1)\right) .
$$

On obtient :

$$
\begin{aligned}
q(s) & =\frac{2(2 \pi)^{-\left(\lambda_{1}+s\right)} 2^{\lambda_{1}+s-1} \pi^{-\frac{1}{2}} \Gamma\left(\frac{1}{2}\left(\lambda_{1}+s\right)\right) \Gamma\left(\frac{1}{2}\left(\lambda_{1}+s+1\right)\right)}{\pi^{-\frac{1}{2}\left(\lambda_{1}+\alpha_{1}+s\right)} \pi^{-\frac{1}{2}\left(\lambda_{2}+\alpha_{2}+s\right)} \Gamma\left(\frac{1}{2}\left(\lambda_{1}+\alpha_{1}+s\right)\right) \Gamma\left(\frac{1}{2}\left(\lambda_{2}+\alpha_{2}+s\right)\right)} \\
& =\pi^{\frac{1}{2}\left(\lambda_{2}-\lambda_{1}+\alpha_{1}+\alpha_{2}-1\right)} \frac{\Gamma\left(\frac{1}{2}\left(\lambda_{1}+s\right)\right) \Gamma\left(\frac{1}{2}\left(\lambda_{1}+s+1\right)\right)}{\Gamma\left(\frac{1}{2}\left(\lambda_{1}+\alpha_{1}+s\right)\right) \Gamma\left(\frac{1}{2}\left(\lambda_{2}+\alpha_{2}+s\right)\right)} .
\end{aligned}
$$

Comme $\left(\lambda_{1}-\lambda_{2}\right)$ est un entier positif de parité $\alpha_{1}+\alpha_{2}+1$, on conclut en utilisant la formule $\Gamma(z+1)=z \Gamma(z)$ plusieurs fois. Deux cas sont possibles :

- Si $\alpha_{1}=0$, alors $\frac{1}{2}\left[\left(\lambda_{1}+s+1\right)-\left(\lambda_{2}+\alpha_{2}+s\right)\right]=\frac{1}{2}\left[\lambda_{1}-\lambda_{2}+1-\alpha_{2}\right]$ est entier strictement positif et

$$
q(s)=\pi^{\frac{1}{2}\left(\lambda_{2}-\lambda_{1}+\alpha_{1}+\alpha_{2}-1\right)} \frac{1}{2}\left(\lambda_{1}+s+1\right) \frac{1}{2}\left(\lambda_{1}+s-1\right) \cdots \frac{1}{2}\left(\lambda_{2}+\alpha_{2}+s+2\right) .
$$

- Si $\alpha_{1}=1$, alors $\frac{1}{2}\left[\left(\lambda_{1}+s\right)-\left(\lambda_{2}+\alpha_{2}+s\right)\right]=\frac{1}{2}\left[\lambda_{1}-\lambda_{2}-\alpha_{2}\right]$ est entier strictement positif et

$$
q(s)=\pi^{\frac{1}{2}\left(\left(\lambda_{2}-\lambda_{1}+\alpha_{1}+\alpha_{2}-1\right)\right.} \frac{1}{2}\left(\lambda_{1}+s\right) \frac{1}{2}\left(\lambda_{1}+s-2\right) \cdots \frac{1}{2}\left(\lambda_{2}+\alpha_{2}+s+2\right) .
$$


Calculs de divisibilité pour $\mathrm{GL}(2, \mathbb{R})$ dans le cas d'une représentation $r$ quelconque. - Soit $r$ une représentation de $\mathrm{GL}(2, \mathbb{C})$ de dimension finie. Pour calculer les fonctions $L$ associées aux représentations $\pi_{[p, q]}$ et $\pi_{\left(\lambda_{1}, \alpha_{1}, \lambda_{2}, \alpha_{2}\right)}$, il faut décomposer les représentations du groupe de Weil $r \circ\left(\chi_{\lambda_{1}, \alpha_{1}}+\chi_{\lambda_{2}, \alpha_{2}}\right)$ et $r \circ \theta_{p, q}$.

Soit $r_{\nu, \mu}$ la représentation de poids dominant (par rapport au groupe de Borel triangulaire supérieur) $\nu, \mu$ (où $\nu$ et $\mu$ sont des entiers et $\nu-\mu$ est positif ou nul). Cette représentation se réalise comme le produit tensoriel de $(\text { det })^{\nu}$ et de la représentation régulière de $\mathrm{GL}(2, \mathbb{C})$ dans l'ensemble des polynômes homogènes en deux variables de degré $(\nu-\mu)$. On obtient alors la décomposition

$$
r_{\nu, \mu} \circ\left(\chi_{\lambda_{1}, \alpha_{1}}+\chi_{\lambda_{2}, \alpha_{2}}\right)=\sum_{i=0}^{\nu-\mu} \chi_{\lambda^{i}, \alpha^{i}}
$$

où

$$
\lambda^{i}=\lambda_{1}(\nu-i)+\lambda_{2}(\mu+i), \quad \alpha^{i} \equiv \alpha_{1}(\nu-i)+\alpha_{2}(\mu+i) .
$$

L'autre décomposition dépend de la parité de $(\nu-\mu)$ :

- $\mathrm{Si}(\nu-\mu)$ est pair, la décomposition est

$$
r_{\nu, \mu} \circ \theta_{p, q}=\sum_{j=0}^{\frac{1}{2}(\nu-\mu)-1} \theta_{p^{j}, q^{j}}+\chi_{\lambda, \alpha}
$$

où

$$
\begin{aligned}
p^{j}=(\nu-j) p+(\mu+j) q, & q^{j}=(\mu+j) p+(\nu-j) q, \\
\lambda=(p+q) \frac{1}{2}(\nu+\mu)=\lambda^{\frac{1}{2}(\nu-\mu)}, & \alpha \equiv(p-q) \frac{1}{2}(\nu+\mu)+\nu .
\end{aligned}
$$

- $\mathrm{Si}(\nu-\mu)$ est impair, la décomposition est

$$
r_{\nu, \mu} \circ \theta_{p, q}=\sum_{j=0}^{\frac{1}{2}(\nu-\mu-1)} \theta_{p^{j}, q^{j}}
$$

où

$$
p^{j}=(\nu-j) p+(\mu+j) q, \quad q^{j}=(\mu+j) p+(\nu-j) q .
$$

Supposons que $\pi_{\left(\lambda_{1}, \alpha_{1}, \lambda_{2}, \alpha_{2}\right)} \prec \pi_{[p, q]}$ pour le $L$ préordre de Bruhat, c'est-à-dire que $\left(\lambda_{1}-\lambda_{2}\right)$ est entier non nul et $\lambda_{1}-\lambda_{2} \equiv \alpha_{1}+\alpha_{2}+1 \bmod 2$,

TOME $122-1994-\mathrm{N}^{\circ} 1$ 
et que $p=\lambda_{1}, q=\lambda_{2}$. On peut bien supposer que $\left(\lambda_{1}-\lambda_{2}\right)$ est entier strictement positif. Le quotient $q(s)$ des fonctions $L$ est alors :

- Cas $(\nu-\mu)$ pair :

$$
q(s)=\frac{\prod_{i=0}^{\frac{1}{2}(\nu-\mu)-1} \Gamma\left(s+\lambda^{i}\right) \Gamma\left(\frac{1}{2}\left(s+\lambda^{\frac{1}{2}(\nu-\mu)}+\alpha\right)\right)}{\prod_{i=0}^{\nu-\mu} \Gamma\left(\frac{1}{2}\left(s+\lambda^{i}+\alpha^{i}\right)\right)} .
$$

Appliquons la formule de duplication de la fonction $\Gamma$. Il vient

$$
q(s)=\frac{\prod_{i=0}^{\frac{1}{2}(\nu-\mu)-1} \Gamma\left(\frac{1}{2}\left(s+\lambda^{i}\right)\right) \Gamma\left(\frac{1}{2}\left(s+\lambda^{i}+1\right)\right) \Gamma\left(\frac{1}{2}\left(s+\lambda^{\frac{1}{2}(\nu-\mu)}+\alpha\right)\right)}{\prod_{i=0}^{\nu-\mu} \Gamma\left(\frac{1}{2}\left(s+\lambda^{i}+\alpha^{i}\right)\right)} .
$$

-. Si $\left(\lambda_{1}-\lambda_{2}\right)$ est impair, alors $\alpha_{1}$ et $\alpha_{2}$ sont de même parité, donc tous les $\alpha^{i}$ sont égaux à 0 . Il faut encore distinguer deux sous-cas :

•. Si $\frac{1}{2}(\nu-\mu)$ est pair, alors $\alpha \equiv 0$ et

$$
q(s)=\frac{\prod_{i=0}^{\frac{1}{2}(\nu-\mu)-1} \Gamma\left(\frac{1}{2}\left(s+\lambda^{i}+1\right)\right)}{\prod_{i=\frac{1}{2}(\nu-\mu)+1}^{\nu-\mu} \Gamma\left(\frac{1}{2}\left(s+\lambda^{i}\right)\right)}
$$

On décompose $q(s)$ en un produit :

$$
\prod_{i=0}^{\frac{1}{2}(\nu-\mu)-1} \frac{\Gamma\left(\frac{1}{2}\left(s+\lambda^{i}+1\right)\right)}{\Gamma\left(\frac{1}{2}\left(s+\lambda^{i+1+\frac{1}{2}(\nu-\mu)}\right)\right)} .
$$

Chacun des termes est un polynôme, parce que

$$
\frac{1}{2}\left[\left(s+\lambda^{i}+1\right)-\left(s+\lambda^{i+1+\frac{1}{2}(\nu-\mu)}\right)\right]=\frac{1}{2}\left[1+\left(\lambda_{1}+\lambda_{2}\right)\left(\frac{1}{2}(\nu-\mu)\right)\right]
$$

est un entier positif, et qu'on peut donc appliquer $\Gamma(z+1)=z \Gamma(z)$ un certain nombre de fois. 
Si $\frac{1}{2}(\nu-\mu)$ est impair, alors $\alpha \equiv 1$. Le quotient s'écrit :

$$
q(s)=\frac{\prod_{i=0}^{\frac{1}{2}(\nu-\mu)} \Gamma\left(\frac{1}{2}\left(s+\lambda^{i}+1\right)\right)}{\prod_{i=\frac{1}{2}(\nu-\mu)}^{\nu-\mu} \Gamma\left(\frac{1}{2}\left(s+\lambda^{i}\right)\right)} .
$$

On décompose $q(s)$ en un produit :

$$
\prod_{i=0}^{\frac{1}{2}(\nu-\mu)} \frac{\Gamma\left(\frac{1}{2}\left(s+\lambda^{i}+1\right)\right)}{\Gamma\left(\frac{1}{2}\left(s+\lambda^{i+\frac{1}{2}(\nu-\mu)}\right)\right)} .
$$

Chacun des termes est un polynôme, parce que

$$
\frac{1}{2}\left[\left(s+\lambda^{i}+1\right)-\left(s+\lambda^{i+\frac{1}{2}(\nu-\mu)}\right)\right]=\frac{1}{2}\left[\left(\lambda_{1}+\lambda_{2}\right)\left(\frac{1}{2}(\nu-\mu)\right)\right]
$$

est un entier positif et qu'on peut donc appliquer $\Gamma(z+1)=z \Gamma(z)$ un certain nombre de fois.

-. Si $\left(\lambda_{1}-\lambda_{2}\right)$ est pair, alors les $\left(\lambda^{i}-\lambda^{j}\right)$ sont tous de même parité et les $\alpha_{i}$ sont de parité $(\nu-i)$. On écrit le quotient de la manière suivante :

$$
\begin{array}{r}
q(s)=\prod_{i=0}^{\frac{1}{2}(\nu-\mu)-1} \frac{\Gamma\left(\frac{1}{2}\left(s+\lambda^{i}\right)\right) \Gamma\left(\frac{1}{2}\left(s+\lambda^{i}+1\right)\right)}{\Gamma\left(\frac{1}{2}\left(s+\lambda^{i}+\alpha^{i}\right)\right) \Gamma\left(\frac{1}{2}\left(s+\lambda^{\nu-\mu-1-i}+\alpha^{\nu-\mu-1-i}\right)\right)} \\
\times \frac{\Gamma\left(\frac{1}{2}(s+\lambda+\alpha)\right)}{\Gamma\left(\frac{1}{2}\left(s+\lambda^{\nu-\mu}+\alpha^{\nu-\mu}\right)\right)} .
\end{array}
$$

On vérifie simplement que $\alpha^{i}$ et $\alpha^{\nu-\mu-1-i}$ ont des parités opposées. Comme on a vu que $\lambda^{i}$ et $\lambda^{j}$ sont de même parité, il est alors clair que chacun des termes

$$
\frac{\Gamma\left(\frac{1}{2}\left(s+\lambda^{i}\right)\right) \Gamma\left(\frac{1}{2}\left(s+\lambda^{i}+1\right)\right)}{\Gamma\left(\frac{1}{2}\left(s+\lambda^{i}+\alpha^{i}\right)\right) \Gamma\left(\frac{1}{2}\left(s+\lambda^{\nu-\mu-1-i}+\alpha^{\nu-\mu-1-i}\right)\right)}
$$

est un polynôme. Pour le dernier terme, on constate que $\alpha$ est de même parité que $\alpha^{\nu-\mu}$, et donc

$$
\frac{1}{2}\left[\left(s+\lambda^{\frac{1}{2}(\nu-\mu)}+\alpha\right)-\left(s+\lambda^{\nu-\mu}+\alpha^{\nu-\mu}\right)\right]
$$

est un entier.

TOME $122-1994-\mathrm{N}^{\circ} 1$ 
- Cas $(\nu-\mu)$ impair :

$$
q(s)=\frac{\prod_{i=0}^{\frac{1}{2}(\nu-\mu-1)} \Gamma\left(s+\lambda^{i}\right)}{\prod_{i=0}^{\nu-\mu} \Gamma\left(\frac{1}{2}\left(s+\lambda^{i}+\alpha^{i}\right)\right)} .
$$

Appliquons la formule de duplication de la fonction $\Gamma$. Il vient :

$$
\begin{aligned}
q(s) & =\frac{\prod_{i=0}^{\frac{1}{2}(\nu-\mu-1)} \Gamma\left(\frac{1}{2}\left(s+\lambda^{i}\right)\right) \Gamma\left(\frac{1}{2}\left(s+\lambda^{i}+1\right)\right)}{\prod_{i=0}^{\nu-\mu} \Gamma\left(\frac{1}{2}\left(s+\lambda^{i}+\alpha^{i}\right)\right)} \\
& =\prod_{i=0}^{\frac{1}{2}(\nu-\mu-1)} \frac{\Gamma\left(\frac{1}{2}\left(s+\lambda^{i}\right)\right) \Gamma\left(\frac{1}{2}\left(s+\lambda^{i}+1\right)\right)}{\Gamma\left(\frac{1}{2}\left(s+\lambda^{i}+\alpha^{i}\right)\right) \Gamma\left(\frac{1}{2}\left(s+\lambda^{\nu-\mu-i}+\alpha^{\nu-\mu-i}\right)\right)} .
\end{aligned}
$$

Comme

$$
\left(\lambda^{i}+\alpha^{i}\right)-\left(\lambda^{\nu-\mu-i}+\alpha^{\nu-\mu-i}\right) \equiv(\nu-\mu-2 i)\left(\lambda_{1}-\lambda_{2}+\alpha_{1}-\alpha_{2}\right)
$$

est impair, on a alors soit $\alpha^{i}=0$ et

$$
\frac{1}{2}\left[\left(s+\lambda^{i}+1\right)-\left(\lambda^{\nu-\mu-i}+\alpha^{\nu-\mu-i}\right)\right]
$$

est un entier positif, soit $\alpha^{i}=1$ et

$$
\frac{1}{2}\left[\left(s+\lambda^{i}\right)-\left(\lambda^{\nu-\mu-i}+\alpha^{\nu-\mu-i}\right)\right]
$$

est un entier positif. Dans les deux cas, chacun des termes

$$
\frac{\Gamma\left(\frac{1}{2}\left(s+\lambda^{i}\right)\right) \Gamma\left(\frac{1}{2}\left(s+\lambda^{i}+1\right)\right)}{\Gamma\left(\frac{1}{2}\left(s+\lambda^{i}+\alpha^{i}\right)\right) \Gamma\left(\frac{1}{2}\left(s+\lambda^{\nu-\mu-i}+\alpha^{\nu-\mu-i}\right)\right)}
$$

est un polynôme. Le résultat est donc démontré.

Le cas de $G=\mathrm{GL}(3, \mathbb{R})$. — Nous faisons également le calcul complètement dans ce cas, parce qu'il est instructif et qu'il nous permettra de mieux comprendre le calcul fait ci-dessus pour $\operatorname{GL}(2, \mathbb{R})$ et la représentation adjointe $r$. 
Classification de Langlands et suites de composition des représentations standard. - Les représentations standard de GL $(3, \mathbb{R})$ sont de deux types :

- D'une part, les séries principales, induites à partir d'un parabolique minimal $P=M A N$, où $M A$ est le groupe de Cartan habituel : $M$ est isomorphe à $\{-1,1\}^{3}$ et $A$ est isomorphe à $\mathbb{R}^{+3}$. La représentation standard $\tau\left(\alpha_{1}, \lambda_{1}, \alpha_{2}, \lambda_{2}, \alpha_{3}, \lambda_{3}\right)$ est l'induite unitaires à partir du caractère

$$
\left(\begin{array}{ccc}
x_{1} & 0 & 0 \\
0 & x_{2} & 0 \\
0 & 0 & x_{3}
\end{array}\right) \longmapsto\left(\frac{x_{1}}{\left|x_{1}\right|}\right)^{\alpha_{1}}\left|x_{1}\right|^{\lambda_{1}}\left(\frac{x_{2}}{\left|x_{2}\right|}\right)^{\alpha_{2}}\left|x_{2}\right|^{\lambda_{2}}\left(\frac{x_{3}}{\left|x_{3}\right|}\right)^{\alpha_{3}}\left|x_{3}\right|^{\lambda_{3}} .
$$

Ces représentations correspondent dans la classifications de Langlands aux représentations de $W_{\mathbb{R}}$ qui sont sommes de trois caractères

$$
\chi_{\lambda_{1}, \alpha_{1}}+\chi_{\lambda_{2}, \alpha_{2}}+\chi_{\lambda_{3}, \alpha_{3}} .
$$

- D'autre part, il y a les représentations induites à partir de sous groupes paraboliques dont la partie de Levi $M A$ est de la forme

$$
\left(\begin{array}{ccc}
* & * & 0 \\
* & * & 0 \\
0 & 0 & x_{3}
\end{array}\right)
$$

On considère une série discrète de $\operatorname{GL}(2, \mathbb{R}) \pi_{[p, q]}$, et la représentation standard $\tau_{[p, q], \lambda, \alpha}$ est induite à partir de la représentation de $M A N$ :

$$
\left(\begin{array}{ccc}
a & b & u \\
c & d & v \\
0 & 0 & x_{3}
\end{array}\right) \longmapsto \pi_{[p, q]}\left(\left(\begin{array}{ll}
a & b \\
c & d
\end{array}\right)\right)\left(\frac{x_{3}}{\left|x_{3}\right|}\right)^{\alpha}\left|x_{3}\right|^{\lambda} .
$$

Ces représentations correspondent dans la classification de Langlands à la représentation de $W_{\mathbb{R}}$ somme de $\theta p, q$ et $\chi_{\lambda, \alpha}$.

On donne d'abord la suite de composition de $\tau_{[p, q], \lambda, \alpha}$ dans le cas non singulier. Soit $H=T A$ le sous-groupe de Cartan $\theta$-stable de $G$ où $T$ est le sous-groupe de Cartan compact de $M$ formé des matrices

$$
\left(\begin{array}{ccc}
\cos \phi & \sin \phi & 0 \\
-\sin \phi & \cos \phi & 0 \\
0 & 0 & \varepsilon
\end{array}\right)
$$

où $\phi \in \mathbb{R} / 2 \pi \mathbb{Z}, \epsilon= \pm 1$ et $A$ est l'ensemble des matrices

$$
\left(\begin{array}{lll}
a & 0 & 0 \\
0 & a & 0 \\
0 & 0 & b
\end{array}\right)
$$

TOME $122-1994-\mathrm{N}^{\circ} 1$ 
avec $a, b \in \mathbb{R}^{+}$. La sous-algèbre de Cartan correspondante est l'ensemble des matrices

$$
\left(\begin{array}{ccc}
a & -t & 0 \\
t & a & 0 \\
0 & 0 & b
\end{array}\right)
$$

et on peut prendre pour base du système de racines $\Delta\left(\mathfrak{g}_{\mathbb{C}}, \mathfrak{h}_{\mathbb{C}}\right)$ l'ensemble $B=\left\{\alpha_{1}, \alpha_{2}\right\}$ où $\alpha_{1}=2 i t$ et $\alpha_{2}=-i t+a-b$. On a $\theta\left(\alpha_{1}\right)=\alpha_{1}$ et $\theta\left(\alpha_{2}\right)=-\alpha_{1}-\alpha_{2}$, donc $\alpha_{1}$ est imaginaire non compacte, alors que $\alpha_{2}$ est complexe. La racine restreinte $\alpha_{2 \mid \mathfrak{a}_{1}}$ est de multiplicité 2 .

On applique [SV] et [S] pour calculer la suite de composition de $\tau_{[p \cdot q], \lambda, \alpha}$. Dans leurs notations, $\tau_{[p \cdot q], \lambda, \alpha}$ est la représentation associée au caractère régulier $\gamma$ de $T A$ défini par :

$$
\left(\begin{array}{ccc}
\cos \phi & \sin \phi & 0 \\
-\sin \phi & \cos \phi & 0 \\
0 & 0 & \epsilon
\end{array}\right)\left(\begin{array}{lll}
a & 0 & 0 \\
0 & a & 0 \\
0 & 0 & b
\end{array}\right) \longmapsto \exp (i \phi(p-q)) a^{p+q} \epsilon^{\alpha} b^{\lambda}
$$

Supposons d'abord que $\gamma$, considéré comme un élément de $\mathfrak{h}^{*}$, est régulier. On peut supposer que $\left\langle\gamma, \alpha_{1}^{\vee}\right\rangle=p-q$ est un entier strictement positif. La proposition 6.1 de $[\mathrm{SV}]$ entraîne, dans ce cas-ci, que $\tau_{[p, q], \lambda, \alpha}$ est réductible seulement si $\left\langle\gamma, \alpha_{2}^{v}\right\rangle=q-\lambda$ est entier strictement positif ou si $\left\langle\gamma,-\left(\alpha_{1}+\alpha_{2}\right)^{\vee}\right\rangle=\lambda-p$ est un entier strictement positif.

Si $(q-\lambda)$ est un entier strictement positif, par [SV, th. 6.9], un sous-quotient de $\tau_{[p, q], \lambda, \alpha}$ est $\tau_{[p, \lambda], q, \alpha^{\prime}}$ où $\alpha^{\prime}$ est congru à $\alpha+(q-\lambda)$ modulo 2. L'application de la proposition 6.1 de $[\mathrm{SV}]$ montre que $\tau_{[p, \lambda], q, \alpha^{\prime}}$ est irréductible.

Enfin, en utilisant la classification de Langlands, et la proposition 8.2.9 de $[\mathrm{V}]$, on voit que la suite de composition de $\tau_{[p, q], \lambda, \alpha}$ a deux termes : $\pi_{[p, q], \lambda, \alpha}$ et $\tau_{[p, \lambda], q, \alpha^{\prime}}=\pi_{[p, \lambda], q, \alpha^{\prime}}$

De manière analogue, lorsque $(\lambda-p)$ est un entier positif, on montre que la suite de composition de $\tau_{[p, q], \lambda, \alpha}$ a deux termes : $\pi_{[p, q], \lambda, \alpha}$ et $\tau_{[\lambda, q], p, \alpha^{\prime \prime}}=\pi_{[\lambda, q], p, \alpha^{\prime \prime}}$ avec $\alpha^{\prime \prime}$ congru à $\alpha+p-\lambda$ modulo 2 .

Dans le cas singulier, la représentation $\tau_{[p, q], \lambda, \alpha}$ est toujours irréductible.

Pour déterminer la suite de composition de la série principale, les réductions proviennent des réductions dans $G L(2, \mathbb{R})$, et de la suite de composition que l'on vient de calculer. Nous ne traitons que le cas régulier, où il faut distinguer les cas suivants.

- Cas 1. - Aucun des $\left(\lambda_{i}-\lambda_{j}\right)$ n'est entier de parité $\alpha_{i}+\alpha_{j}+1$. Alors $\tau_{\lambda_{1}, \alpha_{1}, \lambda_{2}, \alpha_{2}, \lambda_{3}, \alpha_{3}}$ est irréductible. 
- Cas 2. - Un des $\left(\lambda_{i}-\lambda_{j}\right)$ est entier de parité $\alpha_{i}+\alpha_{j}+1$ et les autres $\left(\lambda_{i}-\lambda_{j}\right)$ ne sont pas entiers. Alors $\tau_{\lambda_{1}, \alpha_{1}, \lambda_{2}, \alpha_{2}, \lambda_{3}, \alpha_{3}}$ a une suite de composition à deux termes : prenons par exemple le cas $\left(\lambda_{1}-\lambda_{2}\right)$ entier de parité $\alpha_{1}+\alpha_{2}+1$, alors la suite de composition comprend $\pi_{\lambda_{1}, \alpha_{1}, \lambda_{2}, \alpha_{2}, \lambda_{3}, \alpha_{3}}$ et $\pi_{\left[\nu_{2}, \nu_{3}\right], \nu_{1}, \alpha_{1}}$.

- Cas 3. - Deux des $\left(\lambda_{i}-\lambda_{j}\right)$ sont entiers de parité $\alpha_{i}+\alpha_{j}+1$ et l'autre est entier de parité $\alpha_{i}+\alpha_{j}$. Prenons le cas $\lambda_{1}-\lambda_{2} \equiv \alpha_{1}+\alpha_{2}+1$, $\lambda_{2}-\lambda_{3} \equiv \alpha_{2}+\alpha_{3}+1$. Alors la suite de composition de $\tau_{\lambda_{1}, \alpha_{1}, \lambda_{2}, \alpha_{2}, \lambda_{3}, \alpha_{3}}$ comprend les représentations suivantes : $\pi_{\lambda_{1}, \alpha_{1}, \lambda_{2}, \alpha_{2}, \lambda_{3}, \alpha_{3}}, \pi_{\left[\lambda_{1}, \lambda_{2}\right], \lambda_{3}, \alpha_{3}}$, $\pi_{\left[\lambda_{2}, \lambda_{3}\right], \lambda_{1}, \alpha_{1}}$ et $\pi_{\left[\lambda_{1}, \lambda_{3}\right], \lambda_{2}, \alpha_{2}^{\prime}}$, où $\alpha_{2}^{\prime} \equiv \alpha_{1}+\left(\lambda_{1}-\lambda_{2}\right) \equiv \alpha_{3}+\left(\lambda_{2}-\lambda_{3}\right)$.

Calculs de divisibilité des fonctions $L$. - D'après l'étape de réduction au cas des représentations induites à partir de paraboliques de rang réel 1 , le seul calcul à faire est celui concernant la série de composition de $\tau_{[p, q], \lambda, \alpha}$. On peut en effet vérifier que les calculs concernant les suites de composition des séries principales sont de deux types : les uns proviennent des calculs déjà faits pour $\mathrm{GL}(2, \mathbb{R})$; les autres sont ceux relatifs à $\tau_{[p, q], \lambda, \alpha}$.

La fonction $L$ principale associée à $\theta_{p, q}+\chi_{\lambda, \alpha}$ est par définition :

$$
\begin{gathered}
L\left(\theta_{p, q}+\chi_{\lambda, \alpha}\right)=2(2 \pi)^{-(\max (p, q)+s)} \Gamma(\max (p, q)+s) \\
\pi^{-\frac{1}{2}(\lambda+\alpha+s)} \Gamma\left(\frac{1}{2}(\lambda+\alpha+s)\right) \\
=2(2 \pi)^{-(\max (p, q)+s)} \pi^{-\frac{1}{2}(\lambda+\alpha+s)} \\
\Gamma(\max (p, q)+s) \Gamma\left(\frac{1}{2}(\lambda+\alpha+s)\right) .
\end{gathered}
$$

Dans le cas $(q-\lambda)$ entier strictement positif, il faut donc calculer le quotient :

$$
\begin{aligned}
q(s) & =(2 \pi)^{-\max (p, \lambda)+\max (p, q)} \pi^{\frac{1}{2}\left(-q-\alpha^{\prime}+\lambda+\alpha\right)} \\
& \frac{\Gamma(\max (p, \lambda)+s) \Gamma\left(\frac{1}{2}\left(q+\alpha^{\prime}+s\right)\right)}{\Gamma(\max (p, q)+s) \Gamma\left(\frac{1}{2}(\lambda+\alpha+s)\right)} \\
& =\pi^{\frac{1}{2}\left(\lambda-q+\alpha-\alpha^{\prime}\right)} \frac{\left.\Gamma(p+s) \Gamma\left(\frac{1}{2}\left(q+\alpha^{\prime}+s\right)\right)\right)}{\Gamma(p+s) \Gamma\left(\frac{1}{2}(\lambda+\alpha+s)\right)} \\
& =\pi^{\frac{1}{2}\left(\lambda-q+\alpha-\alpha^{\prime}\right)} \frac{\Gamma\left(\frac{1}{2}\left(q+\alpha^{\prime}+s\right)\right)}{\Gamma\left(\frac{1}{2}(\lambda+\alpha+s)\right)} .
\end{aligned}
$$

Soit $\beta \in\{0,1\}$ congru à $(q-\lambda)$ modulo 2. On a $\alpha^{\prime}=(\alpha-\beta)^{2}=$ $\alpha+\beta-2 \alpha \beta$, donc $q+\alpha^{\prime}+s-\lambda-\alpha-s=q-\lambda+\beta-2 \alpha \beta$ est un entier pair et positif ou nul. On conclut donc que

$$
q(s)=\pi^{\frac{1}{2}(\lambda-q-\beta+2 \alpha \beta)} \frac{1}{2}\left(s+q+\alpha^{\prime}-2\right) \cdots \frac{1}{2}(s+\lambda+\alpha) .
$$

On conclut de même dans le cas $(\lambda-p)$ entier strictement positif. 
Retour sur le calcul pour $\mathrm{GL}(2, \mathbb{R})$ et la représentation adjointe. Reprenons le calcul fait plus haut concernant $\mathrm{GL}(2, \mathbb{R})$ et une représentation $r$ quelconque dans le cas de la représentation adjointe. Dans ce cas, $r$ est de dimension 3 , et, avec nos notations $\nu=1, \mu=-1$. On a alors :

$$
q(s)=\frac{\Gamma\left(\frac{1}{2}\left(s+\lambda_{0}\right)\right) \Gamma\left(\frac{1}{2}\left(s+\lambda_{0}+1\right)\right) \Gamma\left(\frac{1}{2}\left(s+\left(\lambda_{1}+\lambda_{2}\right) \frac{1}{2}(\nu+\mu)+\alpha\right)\right)}{\Gamma\left(\frac{1}{2}\left(s+\lambda_{0}+\alpha_{0}\right)\right) \Gamma\left(\frac{1}{2}\left(s+\lambda_{1}+\alpha_{1}\right)\right) \Gamma\left(\frac{1}{2}\left(s+\lambda_{2}+\alpha_{2}\right)\right)} .
$$

Les calculs fait plus haut amènent à distinguer entre les cas $\left(\lambda_{1}-\lambda_{2}\right)$ pair et impair - ce qui rend le calcul difficilement compréhensible, bien qu'il soit assez facile dans ce cas. Le transfert explique bien ce qui se passe.

En effet, le transfert de $\operatorname{GL}(2, \mathbb{R})$ à $G L(3, \mathbb{R})$ de la série principale $\tau_{\lambda_{1}, \alpha_{1}, \lambda_{2}, \alpha_{2}}$ par la représentation adjointe est la série principale

$$
\tau_{\lambda_{1}-\lambda_{2}, \beta, 0,0, \lambda_{2}-\lambda_{1}, \beta}
$$

où $\beta \equiv \alpha_{1}-\alpha_{2}$. Le transfert de $\operatorname{GL}(2, \mathbb{R})$ à $\operatorname{GL}(3, \mathbb{R})$ de la série discrète $\pi_{\left[\lambda_{1}, \lambda_{2}\right]}$ est la représentation $\pi_{\left[\lambda_{1}-\lambda_{2}, \lambda_{2}-\lambda_{1}\right], 0, \alpha}$ de $\operatorname{GL}(3, \mathbb{R})$ (la représentation standard correspondante est irréductible), où

$$
\alpha \equiv\left(\lambda_{1}-\lambda_{2}\right) \frac{1}{2}(\nu+\mu)+\nu \equiv 1 \text {. }
$$

D'après ce qu'on a vu, la représentation $\tau_{\lambda_{1}-\lambda_{2}, \beta, 0,0, \lambda_{2}-\lambda_{1}, \beta}$ a une suite de composition avec quatre sous-quotients inéquivalents : en effet, $\beta$ est de parité opposée à celle de $\left(\lambda_{1}-\lambda_{2}\right)$. En dehors du sous-quotient de Langlands, deux sous-quotients 《proviennent» de $\mathrm{GL}(2, \mathbb{R})$, à savoir $\pi_{\left[\lambda_{1}-\lambda_{2}, 0\right], \lambda_{2}-\lambda_{1}, \beta}$ et $\pi_{\left[0, \lambda_{2}-\lambda_{1}\right], \lambda_{1}-\lambda_{2}, \beta}$ et le dernier, $\pi_{\left[\lambda_{1}-\lambda_{2}, \lambda_{2}-\lambda_{1}\right], 0,1}$, provient d'une combinaison d'un phénomène «de rang 1 » issu de $\operatorname{GL}(2, \mathbb{R})$ et d'un phénomène propre à $\mathrm{GL}(3, \mathbb{R})$. C'est ce dernier sous-quotient qui est le transfert de la série discrète.

\section{Démonstration des théorèmes}

Démonstration du théorème 1. - Nous avons donc deux groupes réels $G$ et $G^{\prime}$ vérifiant les hypothèses générales, leurs $L$-groupes ${ }^{\vee} G^{\Gamma}$ et ${ }^{\vee} G^{\prime \Gamma}$. On se donne un $L$-homomorphisme $r$ de ${ }^{\vee} G^{\Gamma}$ dans ${ }^{\vee} G^{\prime \Gamma}$, c'est-à-dire un homomorphisme rationnel tel que le diagramme suivant soit commutatif :

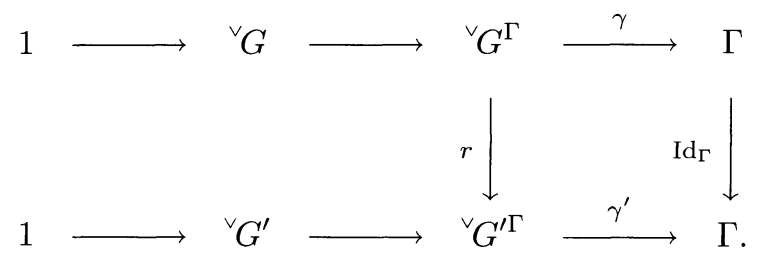


Ceci définit une application $\mathcal{P}(r)$ de $\mathcal{P}(G)$ dans $\mathcal{P}\left(G^{\prime}\right)$ et donc une application $\Phi(r)$ de $\Phi(G / \mathbb{R})$ dans $\Phi\left(G^{\prime} / \mathbb{R}\right)$. Notons de la même lettre l'homomorphisme $r$ de ${ }^{\vee} \mathfrak{g}$ dans ${ }^{\vee} \mathfrak{g}$ ' dérivé de $r$. Soit $\phi \in \mathcal{P}(G)$ associé aux paramètres $(y, \lambda)$. Il est facile de voir que $\mathcal{P}(r)(\phi)$ est associé aux paramètres $(r(y), r(\lambda))$. Un élément semi-simple $\lambda$ étant donné, on vérifie aisément que :

$$
\begin{gathered}
r\left({ }^{\vee} \mathfrak{g}(\lambda)_{n}\right) \subset{ }^{\vee} \mathfrak{g}^{\prime}(r(\lambda))_{n}, \\
r\left({ }^{\vee} \mathfrak{l}(\lambda)\right) \subset{ }^{\vee} \mathfrak{l}^{\prime}(r(\lambda)), \\
r\left({ }^{\vee} \mathfrak{n}(\lambda)\right) \subset{ }^{\vee} \mathfrak{n}^{\prime}(r(\lambda)), \\
r\left({ }^{\vee} \mathfrak{p}(\lambda)\right) \subset{ }^{\vee} \mathfrak{p}^{\prime}(r(\lambda)), \\
r(e(\lambda))=e^{\prime}(r(\lambda)), \\
r\left({ }^{\vee} G(\lambda)\right) \subset{ }^{\vee} G^{\prime}(r(\lambda)), \\
r\left({ }^{\vee} L(\lambda)\right) \subset{ }^{\vee} L^{\prime}(r(\lambda)), \\
r\left({ }^{\vee} N(\lambda)\right) \subset{ }^{\vee} N^{\prime}(r(\lambda)), \\
r\left({ }^{\vee} P(\lambda)\right) \subset{ }^{\vee} P^{\prime}(r(\lambda)) .
\end{gathered}
$$

L'assertion (24) prouve que l'image par $r$ du plateau canonique $\mathcal{F}(\lambda)$ est incluse dans le plateau $\mathcal{F}(r(\lambda))$. Si $\mathcal{F}=\mathcal{F}(\lambda)$, on note $r(\mathcal{F})$ pour le plateau $\mathcal{F}(r(\lambda))$. On déduit donc de $r$ une application, notée $\mathcal{X}(r)$, de $\mathcal{X}\left({ }^{\vee} G^{\Gamma}\right)$ dans $\mathcal{X}\left({ }^{\vee} G^{\prime \Gamma}\right)$ définie par :

$$
\mathcal{X}(r)(y, \mathcal{F})=(r(y), r(\mathcal{F}))
$$

Le diagramme suivant est commutatif :

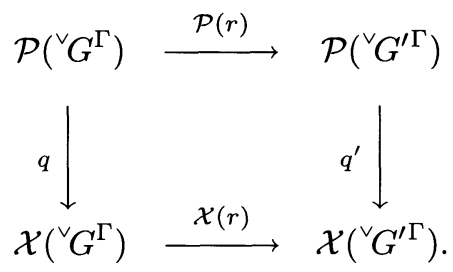

Soit $\mathcal{O}$ une orbite semi-simple dans ${ }^{\vee} \mathfrak{g}$. Son image $r(\mathcal{O})$ est incluse dans une unique orbite semi-simple dans ' $\mathfrak{g}^{\prime}$. Notons $r(\mathcal{O})$ cette orbite. On remarque que $r\left(\mathcal{X}\left(\mathcal{O},{ }^{\vee} G^{\Gamma}\right)\right) \subset \mathcal{X}\left(r(\mathcal{O}),{ }^{\vee} G^{\prime \Gamma}\right)$. Notons $\mathcal{X}(\mathcal{O}, r)$ cette restriction. L'application $\mathcal{C}(r)$ de $\mathcal{C}(\mathcal{O})$ dans $\mathcal{C}(\mathfrak{r}(\mathcal{O}))$ déduite de $r$ est

TOME $122-1994-\mathrm{N}^{\circ} 1$ 
rationnelle. On a en effet le diagramme commutatif :

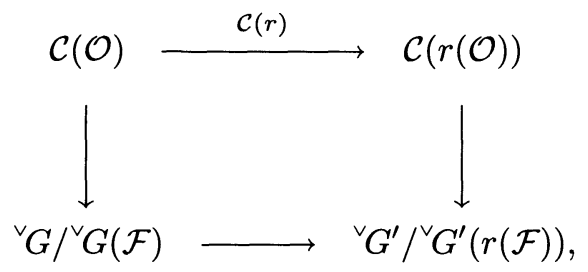

où les identifications verticales sont celles définies dans le paragraphe II, et la flèche du bas est l'application naturellement déduite de $r$. De la même manière, on montre que l'application $\mathcal{F} \mapsto r(\mathcal{F})$ est rationnelle de $\mathcal{P}(\mathcal{O})$ dans $\mathcal{P}(r(\mathcal{O}))$ en regardant le diagramme commutatif :

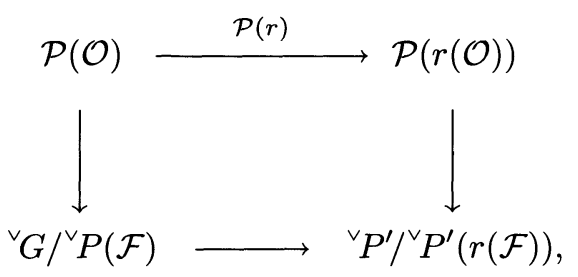

où les identifications verticales sont celles données au paragraphe II, et la flèche du bas celle déduite naturellement de $r$. De ces applications on déduit une application rationnelle du produit fibré $\mathcal{P}(\mathcal{O}) \times_{\mathcal{C}(\mathcal{O})} \mathcal{I}(\mathcal{O})$ dans le produit fibré $\mathcal{P}(r(\mathcal{O})) \times_{\mathcal{C}(r(\mathcal{O}))} \mathcal{I}(r(\mathcal{O}))$ qui n'est autre que $\mathcal{X}(\mathcal{O}, r)$ après les identifications $\mathcal{P}(\mathcal{O}) \times_{\mathcal{C}(\mathcal{O})} \mathcal{I}(\mathcal{O}) \simeq \mathcal{X}\left(\mathcal{O},{ }^{\vee} G^{\Gamma}\right)$ et $\mathcal{P}(r(\mathcal{O})) \times_{\mathcal{C}(r(\mathcal{O}))}$ $\mathcal{I}(r(\mathcal{O})) \simeq \mathcal{X}\left(r(\mathcal{O})^{\vee} G^{\prime \Gamma}\right)$. Cela montre que $\mathcal{X}(\mathcal{O}, r)$ est continue.

Choisissons deux $L$-paquets dans $\Pi(G(\mathbb{R}))$ de représentations irréductibles $\Pi_{S}$ et $\Pi_{S^{\prime}}$ associées aux ${ }^{\vee} G$-orbites $S$ et $S^{\prime}$ dans $\mathcal{X}\left({ }^{\vee} G^{\Gamma}\right)$ tels que $\Pi_{S} \prec \Pi_{S^{\prime}}$ pour le $L$-préordre de Bruhat. D'après la Proposition 2, on a $S \subset \bar{S}^{\prime}$. En particulier, les deux orbites sont incluses dans un même $\mathcal{X}\left(\mathcal{O},{ }^{\vee} G^{\Gamma}\right)$. Par continuité de $\mathcal{X}(\mathcal{O}, r)$, on en déduit que $\mathcal{X}(\mathcal{O}, r)(S) \subset \overline{\mathcal{X}(\mathcal{O}, r)\left(S^{\prime}\right)}$. Le groupe $G^{\prime}(\mathbb{R})$ étant quasi-déployé, la réciproque de la Proposition 2 est vraie pour $G^{\prime}(\mathbb{R})$. Alors

$$
\Pi_{\mathcal{X}(r)(S)} \prec \Pi_{\mathcal{X}(r)\left(S^{\prime}\right)}
$$

pour le préordre de Bruhat dans ${ }^{L} \Pi(G(\mathbb{R}))\left(G^{\prime}(\mathbb{R})\right)$.

Démonstration du théorème 2. - On a démontré le cas particulier des fonctions $L$ principales pour le groupe linéaire au paragraphe 4 . Soit $G$ 
un groupe réel, et soit $r$ une représentation rationnelle de dimension $N$ du $L$-groupe ${ }^{\vee} G^{\Gamma}$ (rappelons que ${ }^{\vee} G^{\Gamma}$ est un groupe algébrique complexe). Notons $\gamma$ l'application canonique de ${ }^{\vee} G^{\Gamma}$ dans le groupe de Galois $\Gamma=\Gamma_{\mathbb{C} \mid \mathbb{R}}$. Nous pouvons considérer l'application $\tilde{r}$ de ${ }^{\vee} G^{\Gamma}$ dans le produit direct $G L(N, \mathbb{C}) \times \Gamma$ définie par

$$
\tilde{r}(g)=(r(g), \gamma(g))
$$

Comme le $L$-groupe de $G L(N, \mathbb{R})$ est le produit direct de $\operatorname{GL}(N, \mathbb{C})$ par le groupe de Galois $\Gamma_{\mathbb{C} \mid \mathbb{R}}$, il nous est loisible de considérer $\tilde{r}$ comme un $L$ homomorphisme de ${ }^{L} G$ dans le $L$-groupe de $G L(N, \mathbb{R})$. Soit $\pi^{G}=\pi^{G}(\phi)$ un élément de $\Pi(G(\mathbb{R}))$ correspondant à $\phi \in \Phi(G / \mathbb{R})$. Par définition, la fonction $L$ de Langlands (qui ne dépend que du $L$-paquet contenant $\pi$ ) $L\left(\pi^{G}, r, s\right)=L(\phi, r, s)$ associée à la représentation $\pi^{G}$ de $G$ et à la représentation rationnelle $r$ est la fonction $L$ d'Artin associée à la représentation $r \circ \phi$ du groupe de Weil $W_{\mathbb{R}}$, et est aussi la fonction $L$ principale (voir $[J])$ associée à la représentation $\pi^{G L(N, \mathbb{R})}(r \circ \phi)$ de $G L(N, \mathbb{R})$.

Comme le groupe $G L(N, \mathbb{R})$ est quasi-déployé, on lui appliquer le théorème 1 . Soit $\pi_{1}$ et $\pi_{2}$ deux $L$-paquets de représentation irréductibles de $G(\mathbb{R})$ telles que $\pi_{1} \prec \pi_{2}$ pour le $L$-préordre de Bruhat dans $\Pi(G(\mathbb{R})$ ). D'après le ThÉoRÈme $1, r\left(\pi_{1}\right) \prec r\left(\pi_{2}\right)$ pour le $L$-préordre de Bruhat dans $\Pi(G L(N, \mathbb{R}))$. Le ThÉORÈme 2 résulte donc du cas particulier déjà démontré.

\section{BIBLIOGRAPHIE}

[AV1] Adams (J.), Vogan (D.). - L-groups, Projective Representations and the Langlands Classification, American J. Math., t. 114, 1992, p. $45-138$.

[AV2] Adams (J.), Vogan (D.). - Lifting of Characters and Harish Chandra's Method of Descent, (preprint).

[ABV] Adams (J.), Barbasch (D.) and Vogan (D.). - The Langlands Classification and Irreducible Characters for Real Reductive Groups, Birkhäuser, 1992.

TOME $122-1994-\mathrm{N}^{\circ} 1$ 
[A1] Andler (M.). - Relationships of Divisibility between Local $L$ Functions Associated to Representations of Complex Reductive Groups, Non Commutative Harmonic Analysis and Lie Groups, Lecture Notes in Math., t. 1243, 1987, p. 1-14.

[A2] Andler (M.). - Transfer and the Bruhat Order, Canadian J. of Math., t. 44, 1992, p. 911-923.

[BM] Barbasch (D.) and Moy (A.). - A Unitarity Criterion for $p$-adic Groups, Inventiones Math., t. 98, 1989, p. 19-37.

[B] Borel (A.). - Automorphic L-Functions. Automorphic Forms, Representations and L-Functions, Proc. Symposia in Pure Mathematics, t. 33, part II, Amer. Math. Soc. Providence, 1979, p. 27-60.

[D] Duflo (M.). - Sur la classification des idéaux primitifs dans l'algèbre enveloppante d'une algèbre de Lie semi-simple, Annals of Math., t. 105, 1977, p. 107-120.

[GJ] Godement (R.) and Jacquet (H.). - Zeta Functions of Simple Lie Algebras, Lecture Notes in Math., t. 260, 1972.

[J] JACQuet (H.). - Principal L-Functions of the Linear Group. Automorphic Forms, Representations and L-Functions, Proc. Symposia in Pure Mathematics, t. 33, part II, Am. Math. Soc., Providence, 1979, p. $63-86$.

[JL] JacQuet (H.) and Langlands (R.P.). - Automorphic Forms on GL(2), Lecture Notes in Math., Springer Heidelberg, t. 114, 1970.

[L] Langlands (R.P.). - On the Classification of Irreducible Representations of Real Reductive Groups, 1973. Representation Theory and Harmonic Analysis on Semi-Simple Lie Groups, Math. Surveys and Monographs, Am. Math. Soc., Providence, t. 31, 1989, p. 101-170.

[R] Rodier (F.). - Decomposition of Principal Series for Reductive $p$ adic Groups and the Langlands Classification, Operator Algebras and Groups Representations, 1984 .

[S] Spen (B.). - The Unitary Dual for $\mathrm{GL}(3, \mathbb{R})$ and $\mathrm{GL}(4, \mathbb{R})$, Math. Annalen, t. 258, 1981, p. 113-133.

[SV] Spen (B.) and Vogan (D.). - Reducibility of Generalized Principal Series Representations, Acta Math., t. 145, 1980, p. 227-299.

[T] Tate (J.). - Number Theoretic Background. Automorphic Forms, Representations and $L$-Functions, Proc. Symposia in Pure Mathematics, Am. Math. Soc., Providence, t. 33, part II, 1979, p. 3-26.

[V1] Vogan (D.). - Representations of Real Reductive Lie Groups. Birkhaüser Boston, 1981.

[V2] Vogan (D.). - The Kazhdan-Lusztig Conjecture for Real Reductive Groups. Representation Theory of Reductive Groups. - Birkhäuser Boston, 1983 . 